\title{
VLA HI Imaging of the brightest spiral galaxies in Coma*
}

\section{The HI Atlas and deep continuum imaging of selected early type galaxies}

\author{
H. Bravo-Alfaro ${ }^{1}$, V. Cayatte ${ }^{2}$, J. H. van Gorkom ${ }^{3}$, and C. Balkowski ${ }^{2}$ \\ 1 Departamento de Astronomía, Universidad de Guanajuato. Apdo. Postal 144, Guanajuato 36000, México \\ 2 Observatoire de Paris DAEC, and UMR 8631, associé au CNRS et à l'Université Paris 7, 92195 Meudon Cedex, \\ France \\ 3 Department of Astronomy, Columbia University, 550 W 120th Street, New York, NY 10027, USA
}

Received 11 July 2001 / Accepted 4 September 2001

\begin{abstract}
In the first paper of this series we used Hi observations of the 19 brightest spirals in Coma to analyze the dynamical state of the cluster. In this paper we present the detailed Hi distribution and kinematics of the spirals that were detected in $\mathrm{HI}$, and radio continuum data for a sample of star forming and post starburst galaxies in Coma. We discuss the importance of ICM-ISM interactions to explain the observed Hi morphology. A rough comparison of observed Hi sizes with predicted Hi sizes from simulations by Abadi et al. (1999) gives reasonable agreement. We use the results on radio continuum emission to estimate the star formation rate in the PSB galaxies we pointed at. The radio continuum emission in the 11 so called post starburst galaxies, identified by Caldwell et al. (1993) in the cluster, is weak. Eight of the 11 were not detected down to a 3 sigma upper limit of $0.6 \mathrm{mJy}$. This sets an upper limit to the star formation rate in these galaxies of less than $0.2 M_{\odot} \mathrm{yr}^{-1}$. The three detected post starburst galaxies have a star formation rate of less than one solar mass per year. Thus none of the post starburst galaxies in Coma are dust enshrouded starbursts.
\end{abstract}

Key words. galaxies: clusters: individual: Coma - radio lines: galaxies

\section{Introduction}

This is our second paper on the imaging of the neutral hydrogen component as a tracer of environmental effects on galaxies in the Coma cluster. Global properties of Coma related with its dynamical state, as derived from our VLA $21 \mathrm{~cm}$ line survey, have been discussed in a previous paper (Bravo-Alfaro et al. 2000, hereafter referred to as Paper I). In the present work we give a complete catalogue of Hi maps, channel maps, and velocity fields (for cases with sufficient resolution) for 19 detected galaxies. We compare the detailed HI distribution and kinematics with model predictions for the fate of $\mathrm{HI}$ in galaxies in a cluster environment. We pay particular attention to a class of so called starburst (SB) and post starburst (PSB) galaxies identified by Caldwell et al. (1993, C93 throughout this paper). For these galaxies we derive star formation rates (SFR) from the radio continuum emission and discuss the implications of current gas content and star formation rate on the evolution of the galaxies.

\footnotetext{
Send offprint requests to: H. Bravo-Alfaro, e-mail: hector@astro.ugto.mx

* Appendix is only available in electronic form at http://www .edpsciences.org
}

Several physical mechanisms have been proposed to play a major role affecting the evolution of galaxies in clusters; these processes can be classified according to three types: (a) interactions between the ICM and the interstellar medium (Gunn \& Gott 1972; Nulsen 1982; Kenney \& Young 1989), (b) interactions with the cluster gravitational field (e.g. Bekki 1999), and (c) galaxy-galaxy interactions (see Icke 1985; Lavery \& Henry 1994; Moore et al. 1996, 1999). In many cases more than one of these processes will be at work, but which mechanism dominates under specific physical conditions and how it depends on the galaxy trajectory through the cluster is still a matter of debate. For instance Solanes et al. (2000) conclude from an observational study of eighteen clusters that ICM-ISM interactions are most important to explain the observed Hi deficiency. In contrast, Moore et al. (1999) suggest in a numerical study that, in combination with ram-pressure stripping, galaxy harassment may convert disk galaxies into S0s. In spite of this debate, the fact that spirals in the central region of clusters like Virgo show smaller Hi than optical disks (Warmels 1988; Cayatte et al. 1990, 1994), is unquestionably associated with the interaction with the ICM. These studies demonstrate the importance of detailed HI imaging of individual galaxies in clusters with a 
range of ICM conditions to assess what gas removal processes are at work.

Another fundamental process linked with the evolution of galaxies in clusters is the triggering and stopping of bursts of star formation (van den Bergh et al. 1990). As the ISM is the raw material from which stars form, the evolution of $\mathrm{HI}$ deficient galaxies and their star formation properties are undoubtedly affected by the interaction with the environment. From statistical analyses on large scales the SFR is known to decrease with increasing density (Dressler et al. 1999), whereas at smaller scales the ICM may produce significant enhancements to the star formation activity in individual galaxies, as shown in clusters like A 1367 (Gavazzi et al. 1995) and Coma (Bothun \& Dressler 1986). Recent work supports different scenarios: (1) On the theoretical side, Bekki (1999) concludes that the tidal gravitational field of a group-cluster merger may trigger a burst of star formation, accounting for the PSB population in clusters like Coma, while Vollmer et al. (2001b) suggest that local enhancement of star formation could be due to re-accretion of gas clouds after a ram pressure stripping event. (2) Observationally, Dressler et al. (1999) find a trend in the position of SB and PSB galaxies around the cluster, making the hot gas environment the best explanation for this phenomenon (see also Poggianti et al. 1999). From a study of spectroscopically selected post starburst galaxies, which turned out to be mostly located in the field, Zabludoff et al. (1996) conclude that in those more isolated galaxies the post starburst phenomenon is most likely caused by galaxy interactions and mergers.

In this paper we give the atlas of our VLA HI imaging of spirals in Coma, showing that the scenario depicted by the neutral hydrogen constitutes strong evidence in favor of ICM-ISM interaction to explain the Hi morphologies and the star formation histories in the cluster. We also present results on the radio continuum emission obtained as a byproduct of the spectral line observations. These results are used to derive the star formation rate in the so called post starburst galaxies to explore the possibility that these are in fact dust enshrouded starbursts as has recently been suggested by Smail et al. (1999) for the intermediate redshift cluster CL0939+4713 (see also Miller \& Owen 2001).

We organize the paper as follows. In Sect. 2 we review the observations. In Sect. 3 we describe Hi observational results of individual detected galaxies, in Sect. 4 we discuss the role of ram pressure affecting galaxies in Coma, and compare Hi observations with numerical simulations of ram pressure stripping by Abadi et al. (1999) and Vollmer et al. (2001b). In Sect. 5 we discuss the PSB galaxies; we compare our Hi mass limits and current star formation rates based on the continuum with those of the more vigorously star forming galaxies and discuss the implications. The results are summarized in Sect. 6. In Appendix A, available in electronic form, we give the complete catalogue of Hi channel maps. Throughout the paper we assume a distance of $70 \mathrm{Mpc}$ to the Coma cluster (with $H_{0}=100 \mathrm{~km} \mathrm{~s}^{-1} \mathrm{Mpc}^{-1}$ ), where an angular size of 1 arcmin corresponds to a linear size of $\sim 20 \mathrm{kpc}$.

\section{Observational results}

Our data consist of $21 \mathrm{~cm}$ line data obtained with the $\mathrm{VLA}^{1}$. Twelve fields within one Abell radius of the Coma cluster (equivalent to $1.2^{\circ}$ ) were observed with the VLA in its $\mathrm{C}$ configuration, and two of them reobserved in D configuration. The observed fields and the distribution of the 19 galaxies detected in Hi around the cluster are shown in Paper I (Figs. 1 and 2). Most of the fields are devoted to the center of Coma and the regions where SB and PSB galaxies had been reported from optical observations. Our velocity resolution is $21 \mathrm{~km} \mathrm{~s}^{-1}$, and $43 \mathrm{~km} \mathrm{~s}^{-1}$ for some of the galaxies observed in both $\mathrm{C}$ and D configurations. The angular resolution ranges between 20 and 35 arcsec. The rms in the final cubes is between 0.35 and $0.40 \mathrm{mJy}$ beam $^{-1}$, except in those fields observed in both $\mathrm{C}$ and $\mathrm{D}$ configurations, reaching rms values as low as $0.20 \mathrm{mJy}$ beam $^{-1}$. Most of the channel maps shown in the Appendix (electronic version) are smoothed in velocity. More details on the Hi observations are given in Paper I.

Eight out of the 19 detected galaxies are projected within $0.5 \mathrm{Mpc}$ from the cluster center (we consider the position of the cD NGC 4874 as the Coma center). These central galaxies lie inside or near the hot ICM as traced by the ROSAT X-ray emission (Briel et al. 1992; Vikhlinin et al. 1997), and most of them are very Hi deficient. The Hi deficiency is measured following Giovanelli \& Haynes (1985), comparing the observed Hi mass with the expected Hi mass of an equivalent isolated spiral. Several galaxies detected in Hi near the center of Coma show Hi truncated disks as well as offsets between the optical and HI distributions (see Sect. 3). In a few cases unexpected differences between optical and Hi velocities are found. Six of the galaxies detected in Hi are reported by Bothun \& Dressler (1986) as having blue disks; five of these are projected inside or near the cluster X-ray emission. None of the PSB galaxies reported by Caldwell et al. $(1993,1997)$ were detected in Hi; we obtain for some of them HI mass upper limits down to $3 \times 10^{7} M_{\odot}$.

Our radio continuum images are obtained as a byproduct of the Hi observations. The images are built by averaging a set of those line free channels in a given cube. All of the Hi detected spirals (except NGC 4907) are also detected in the continuum with flux densities ranging between 2 and 16 mJy (see Paper I).

\section{Results for $\mathrm{H}$ I detected galaxies}

In this section we briefly describe the Hi morphology of detected galaxies, and the remarkable features concerning galaxy dynamics as revealed by the HI.

\footnotetext{
1 The National Radio Astronomy Observatory is operated by Associated Universities, Inc., under cooperative agreement with the National Science Foundation.
} 


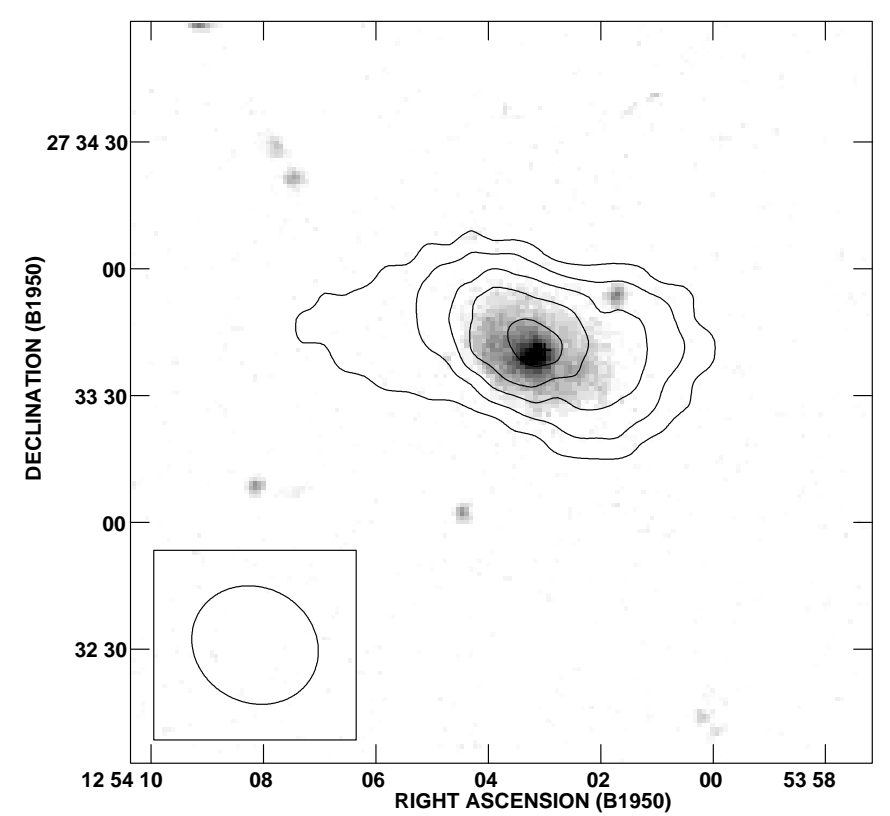

Fig. 1a. Hi density distribution of IC 3913, superposed on a DSS $B$-band gray scale image. The contours are $0.3(2.5 \sigma)$, $1.3,2.7,4.0$, and $5.4 \times 10^{20} \mathrm{~cm}^{-2}$. The $F W H M$ is indicated by the circle, $30.7^{\prime \prime} \times 27.2^{\prime \prime}$.

\section{IC 3913, KUG 1255+275, and Mrk 057}

These three galaxies were detected in the SW of Coma, between $17^{\prime}$ and $25^{\prime}$ south of the cD galaxy NGC 4839 . They display several common features: they are HI rich galaxies with regular gas distributions, where Hi disks extend well beyond the optical (Figs. 1a, 2 and 3a).

Systemic velocities for these galaxies are between $7400 \mathrm{~km} \mathrm{~s}^{-1}$ and $7650 \mathrm{kms}^{-1}$, close to the value of the NGC 4839 group, $7339 \mathrm{~km} \mathrm{~s}^{-1}$ (Colless \& Dunn 1996). Because of their position, lying far from the cluster center and outside the X-ray emission, no strong interaction with the ICM is expected. Although these three galaxies are likely to be part of the infalling NGC 4839 group (Neumann et al. 2001), we see no signs of a gravitational interaction either optically or in Hi or the radio emission. Velocity fields of IC 3913 and Mrk 057 are shown in Figs. $1 \mathrm{~b}$ and $3 \mathrm{~b}$ respectively.

\section{NGC 4848}

This Scd galaxy is one of the most interesting objects in our sample. NGC 4848 is a blue disk galaxy showing a very intriguing Hi distribution (see Fig. 4), as we only detect gas in the northern edge of the galaxy, $19^{\prime \prime}(6.5 \mathrm{kpc})$ NW from the optical center. We are probably missing some Hi flux previously detected by single dish observations in the center of the galaxy (Gavazzi 1989). This object was also observed in $\mathrm{H} \alpha$ by Amram et al. (1992) who reported a double peak in the $\mathrm{H} \alpha$ profile, with the northern $\mathrm{H} \alpha$ peak at the same position than the HI. Interestingly, the brightest HII regions are in the south, where the Hi gas has been totally depleted. The star forming activity is also evidenced by $20 \mathrm{~cm}$ radio continuum emission above a level of $16 \mathrm{mJy}$. This emission shows

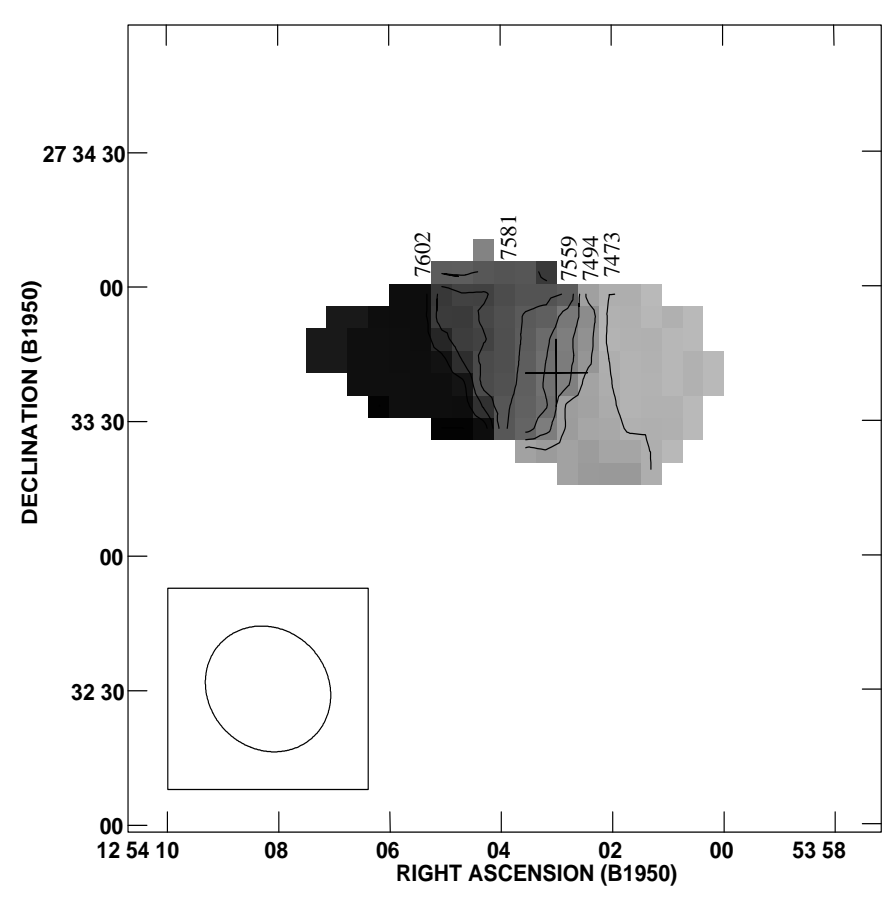

Fig. 1b. Intensity weighted mean velocity field of IC 3913. The optical center of the galaxy is indicated with a cross. The numbers indicate heliocentric velocity in $\mathrm{km} \mathrm{s}^{-1}$. The $F W H M$ is indicated by the circle, $30.7^{\prime \prime} \times 27.2^{\prime \prime}$.

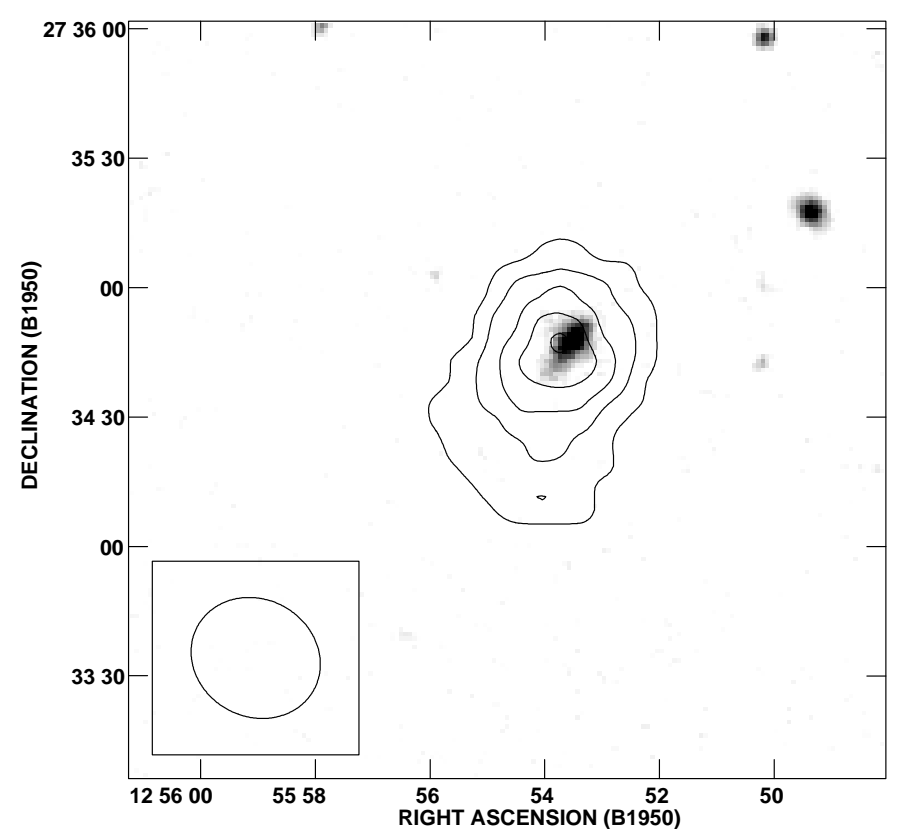

Fig. 2. HI density distribution of KUG $1255+275$, superposed on a DSS $B$-band gray scale image. The contours are 0.3 $(2.5 \sigma), 0.8,1.4,2.0$, and $2.6 \times 10^{20} \mathrm{~cm}^{-2}$. The $F W H M$ is indicated by the circle, $30.7^{\prime \prime} \times 27.2^{\prime \prime}$.

an elongation to the NW which is resolved by the VLA FIRST Survey in two separated sources, one coincident with the optical center of NGC 4848 and a secondary peak $12^{\prime \prime} \mathrm{NW}$, roughly at the same position than the HI. The CO imaging carried out by Vollmer et al. (2001a) shows the maximum of the emission coincident with the optical galaxy center and with the empty Hi zone. A secondary 


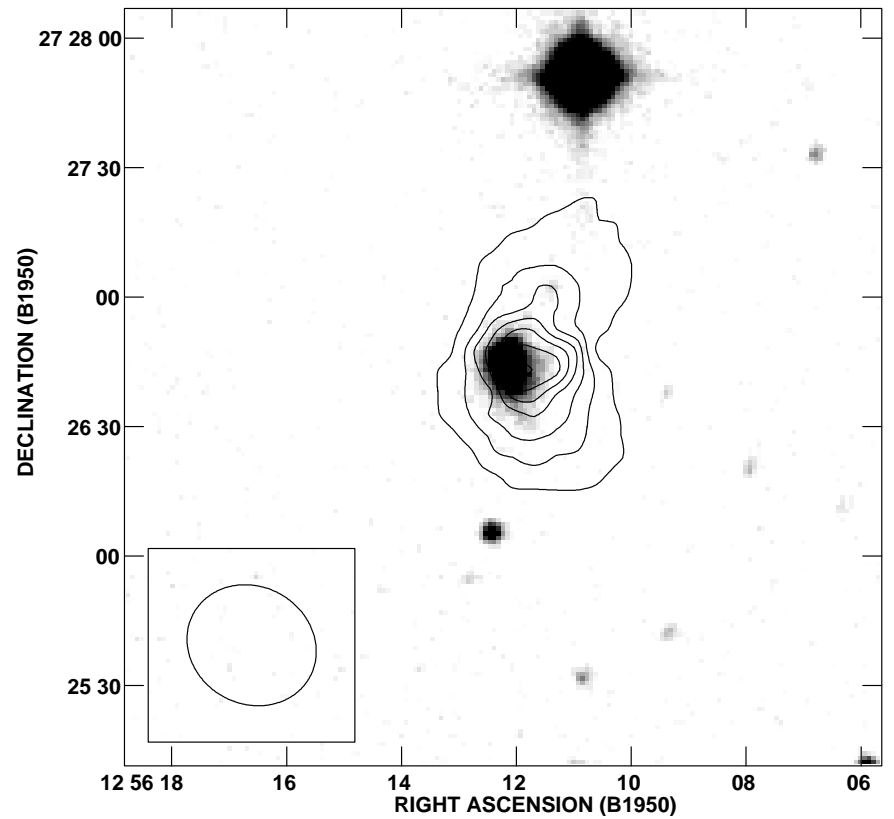

Fig. 3a. Hi density distribution of Mrk 057, superposed on a DSS $B$-band gray scale image. The contours are $0.6(2.5 \sigma), 2.3$, $3.4,4.6,5.7,6.8$ and $8.0 \times 10^{20} \mathrm{~cm}^{-2}$. The $F W H M$ is indicated by the circle, $30.7^{\prime \prime} \times 27.2^{\prime \prime}$.

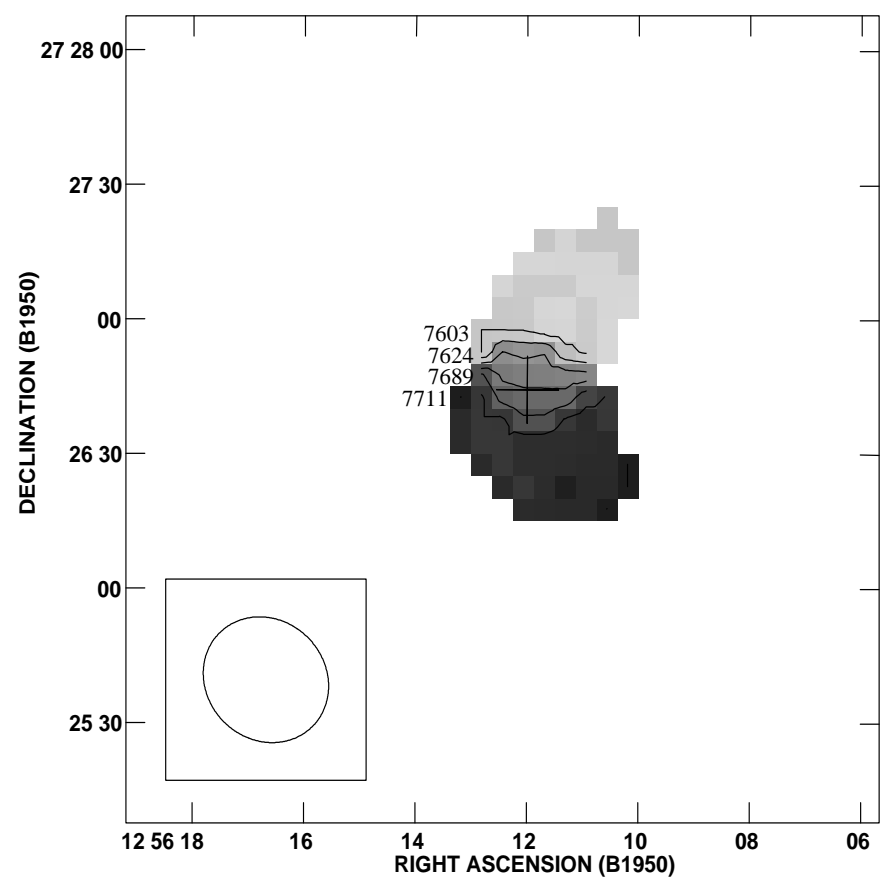

Fig. 3b. Intensity weighted mean velocity field of Mrk 057 . The optical center of the galaxy is indicated with a cross. The numbers indicate heliocentric velocity in $\mathrm{km} \mathrm{s}^{-1}$. The $F W H M$ is indicated by the circle, $30.7^{\prime \prime} \times 27.2^{\prime \prime}$.

CO peak is detected in the NW, roughly coincident with the Hi and radio continuum emission. Lavezzi et al. (1999) reported a normal $M_{\mathrm{H} 2}$ content $\left(2.56 \times 10^{9} M_{\odot}\right)$, which gives a very low fraction of neutral to molecular gas ratio: $M_{\mathrm{HI}} / M_{\mathrm{H} 2} \sim 0.17$ (NGC 4848 is Hi deficient by a factor of around 10).

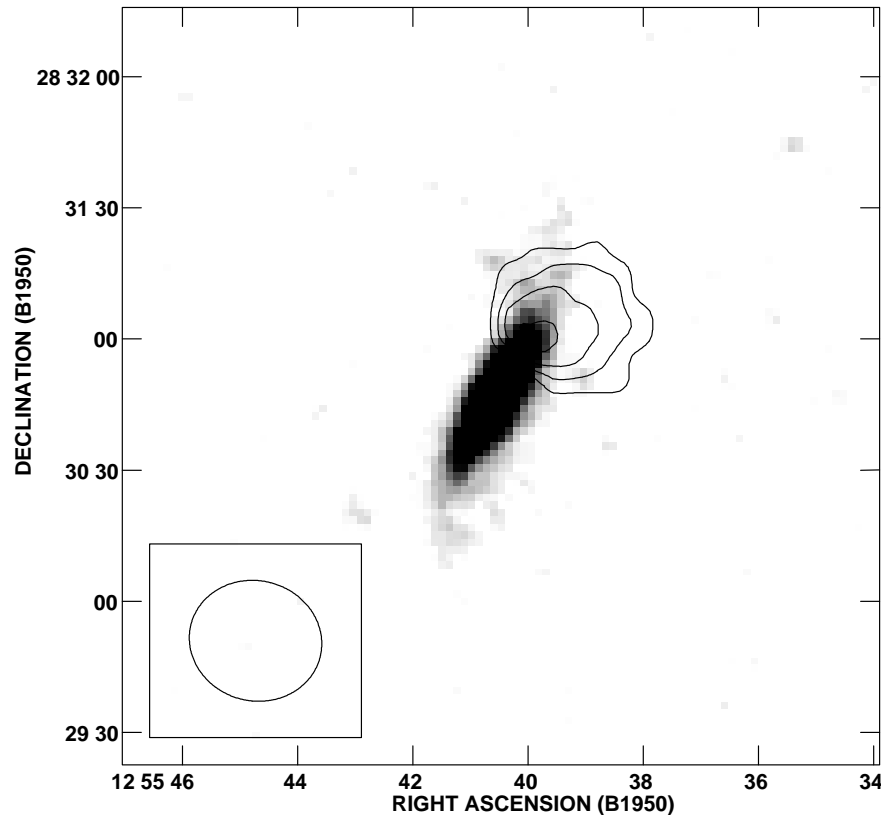

Fig. 4. HI density distribution of NGC 4848, superposed on a DSS $B$-band gray scale image. The contours are $0.3(2.5 \sigma)$, $1.0,1.7$, and $2.3 \times 10^{20} \mathrm{~cm}^{-2}$. The $F W H M$ is indicated by the circle, $30.5^{\prime \prime} \times 27.5^{\prime \prime}$.

Mergers do not account for the Hi morphology or the star formation activity in NGC 4848, because no obvious companion is seen in the DSS optical image (Fig. 4). However, the more detailed B-band CCD imaging by Gavazzi et al. (1990) shows a ring-like structure and blue bright zones in the NW, where the Hi and the secondary peaks of $\mathrm{CO}, \mathrm{H} \alpha$ and $20 \mathrm{~cm}$ radio continuum are found. The hypothesis of a dwarf system crossing the NGC 4848 disk is explored by Vollmer et al. (2001a) but further observations, both optical and higher resolution Hi imaging, are needed to confirm it. $\mathrm{N}$-body simulations by the same authors suggest that NGC 4848 has already gone through the cluster core, $4 \times 10^{8} \mathrm{yr}$ ago, and is now moving away from the cluster. They conclude that re-accretion of some of the stripped gas could explain the star formation burst.

\section{Mrk 58}

This $\mathrm{Sb}$, blue disk galaxy is projected onto the $\mathrm{X}$-ray emission, some $20^{\prime}$ ( $\left.\sim 400 \mathrm{kpc}\right)$ SW of NGC 4874, in the zone lying between the main cluster and the SW group. It is gas deficient by a factor of 3. Its Hi map (Fig. 5) displays a considerably asymmetry, with the gas swept from the NE. The $\mathrm{HI}$ is observed at an offset position of $\sim 12^{\prime \prime}$ (4 kpc) SW from the optical disk. The observed Hi distribution could be explained if we consider the supersonic velocity of Mrk58 relative to the cluster, $1575 \mathrm{~km} \mathrm{~s}^{-1}$ (the sound speed in Coma is estimated around $1460 \mathrm{~km} \mathrm{~s}^{-1}$ by Stevens et al. 1999); under these conditions the interaction with the ICM may produce ram pressure stripping to enhance the density behind the galaxy (Stevens et al. 1999). 


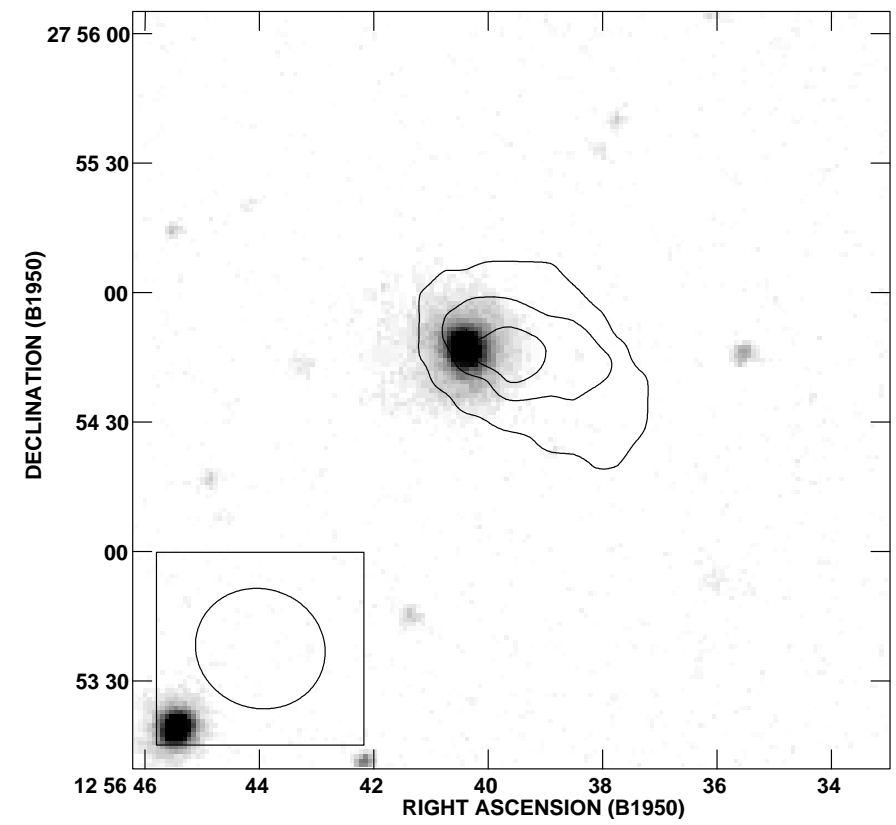

Fig. 5. Hi density distribution of Mrk 058, superposed on a DSS $B$-band gray scale image. The contours are $0.2(2.5 \sigma)$, 0.7 , and $1.1 \times 10^{20} \mathrm{~cm}^{-2}$. The $F W H M$ is indicated by the circle, $30.3^{\prime \prime} \times 27.6^{\prime \prime}$.

\section{CGCG 160-058 and CGCG 160-076}

These late-type spiral galaxies lie in the nearby northern vicinity of Coma, outside the $\mathrm{X}$-ray emission. They are Hi rich galaxies displaying regular gas distributions (Figs. 6a and 7a). As expected, considering their position relative to the cluster, no strong environmental effects are seen: their Hi disks are larger than the optical, and the HI and optical centroids are coincident. Velocity fields are given in Figs. 6b and 7b.

\section{CGCG 160-086}

This Sb, blue disk galaxy, projected onto the SE outskirts of the X-ray emission drawn by ROSAT, was only marginally detected in the present survey. The galaxy is not resolved in HI, as shown in Fig. 8. We report an Hi deficiency by a factor of $\sim 3$, the Hi disk appearing truncated in its periphery, but the low resolution does not allow to get a more conclusive picture.

\section{IC 4040}

This Sdm blue disk galaxy is projected near the very center of Coma, at only 15' (300 kpc) NE from NGC 4874. Its HI distribution (see Fig. 9a) shows the HI contours compressed in the NW, and an offset in position of $9^{\prime \prime}$ ( $\sim 3 \mathrm{kpc}$ ), with the Hi emission SE of the optical galaxy. Our detection confirms the double peak detection reported by Gavazzi (1989) at $7600 \mathrm{~km} \mathrm{~s}^{-1}$ and $7850 \mathrm{~km} \mathrm{~s}^{-1}$. The velocity field for this galaxy is shown in Fig. $9 \mathrm{~b}$.

\section{NGC 4907}

This Sb galaxy was only marginally detected in $\mathrm{HI}$ (Fig. 10). It is Hi deficient by a factor of ten and presents a shrunken HI disk (not resolved by the VLA beam) which is smaller than the optical disk. Two facts make

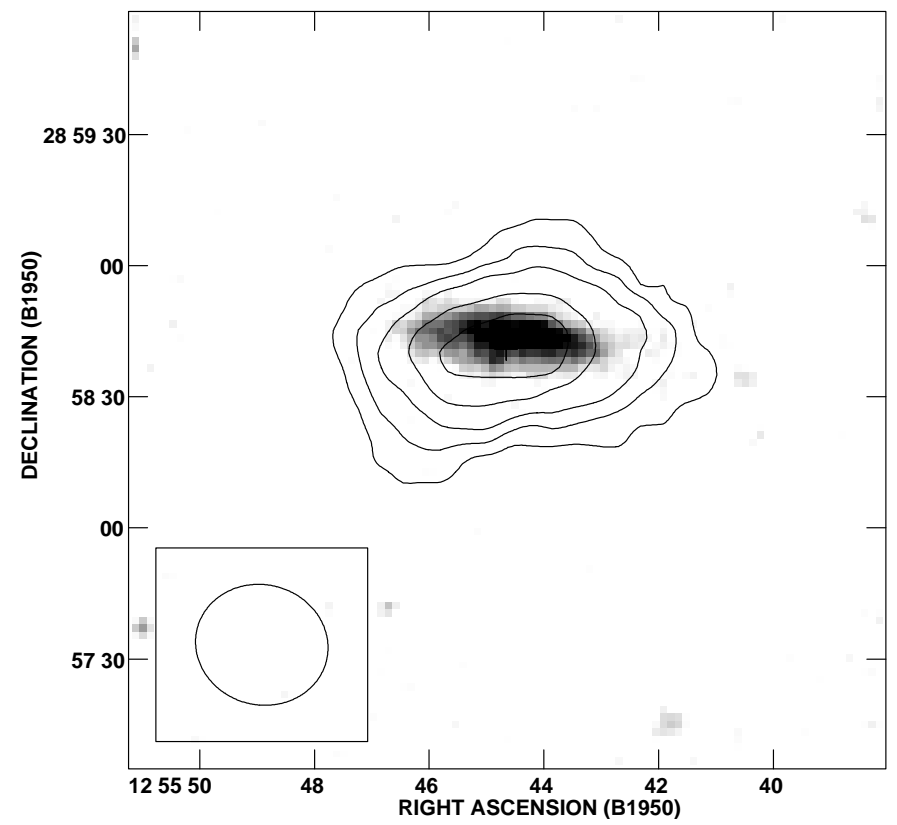

Fig. 6a. Hi density distribution of CGCG 160-058, superposed on a DSS $B$-band gray scale image. The contours are 0.4 $(2.5 \sigma), 1.6,3.2,6.4$, and $9.6 \times 10^{20} \mathrm{~cm}^{-2}$. The $F W H M$ is indicated by the circle, $30.5^{\prime \prime} \times 27.5^{\prime \prime}$.

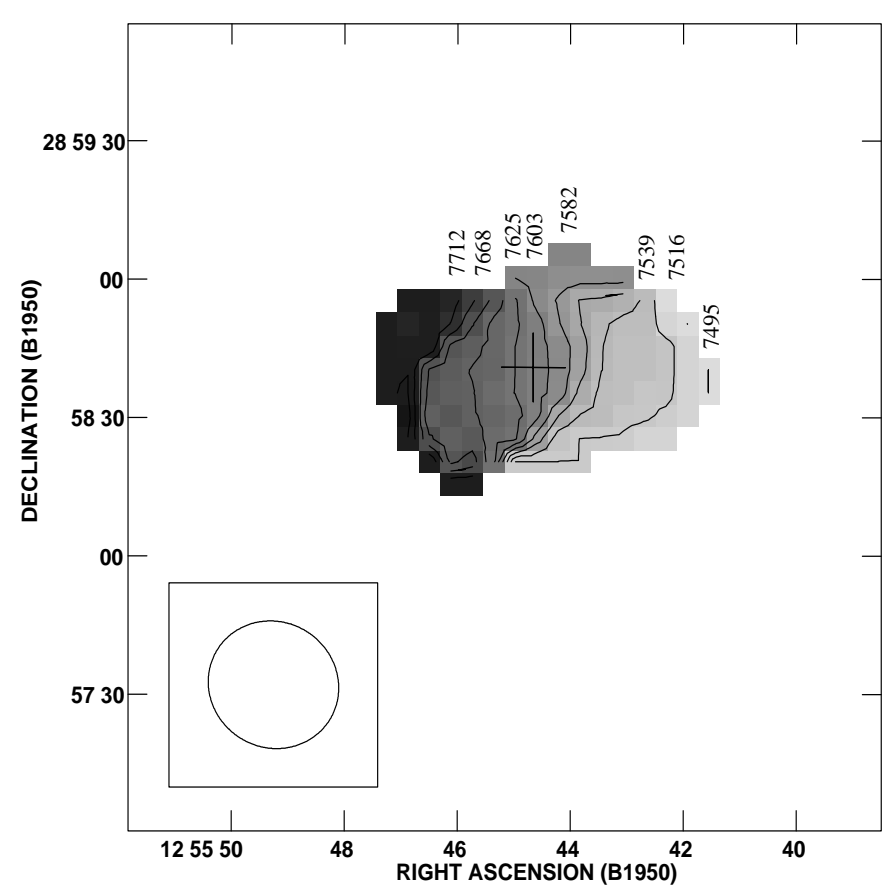

Fig. 6b. Intensity weighted mean velocity field of CGCG 160058. The optical center of the galaxy is indicated with a cross. The numbers indicate heliocentric velocity in $\mathrm{kms}^{-1}$. The $F W H M$ is indicated by the circle, $30.5^{\prime \prime} \times 27.5^{\prime \prime}$.

an ICM-ISM interaction the most likely explanation for this picture: the high radial velocity relative to the cluster $\left(v_{\text {rel }}=1179 \mathrm{kms}^{-1}\right)$, and the projected location of NGC 4907, which puts it in a very high density region, $20^{\prime}$ (400 kpc) NE from the cluster center. 


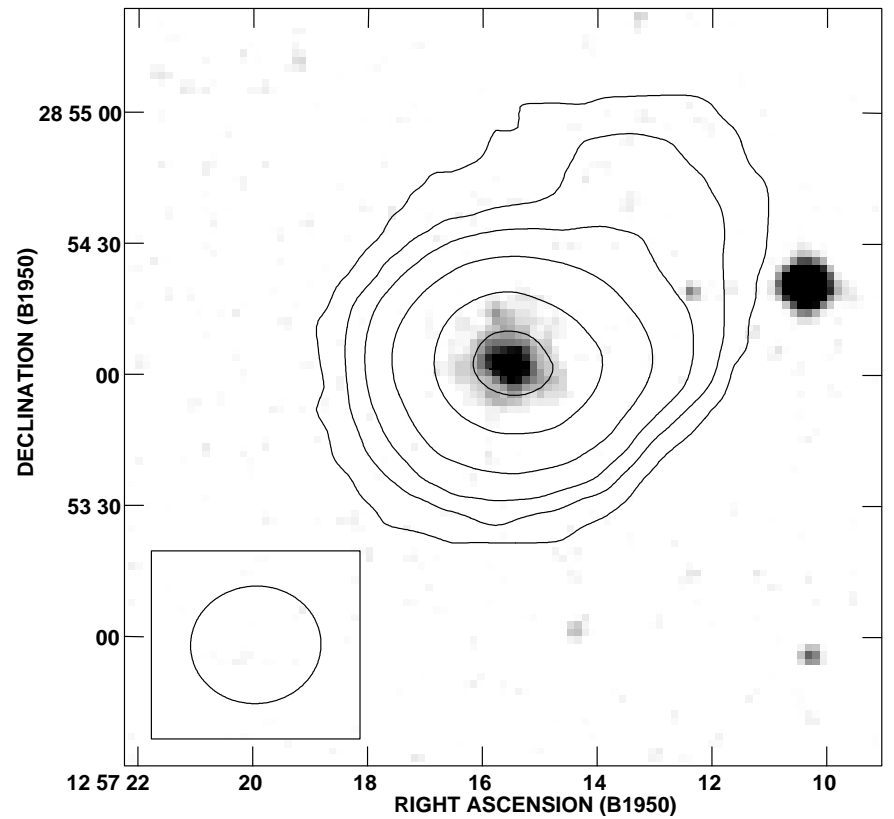

Fig. 7a. Hi density distribution of CGCG 160-076, superposed on a DSS $B$-band gray scale image. The contours are 0.2 $(2.5 \sigma), 0.8,1.6,3.3,6.6$ and $9.9 \times 10^{20} \mathrm{~cm}^{-2}$. The $F W H M$ is indicated by the circle, $29.9^{\prime \prime} \times 26.9^{\prime \prime}$.

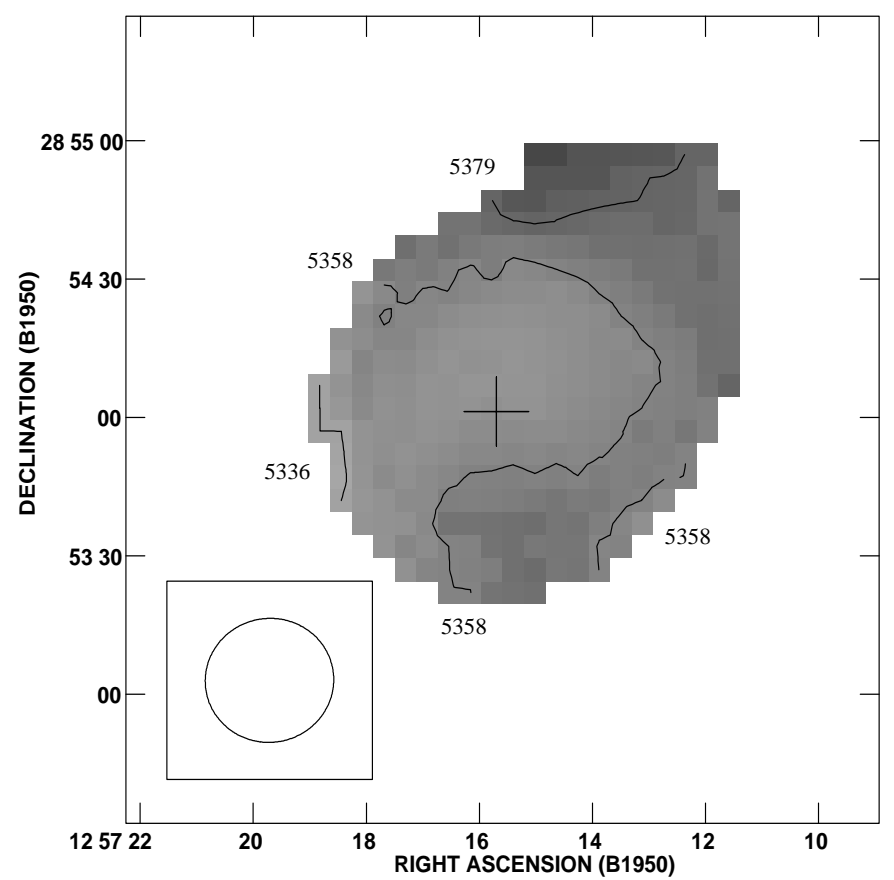

Fig. 7b. Intensity weighted mean velocity field of CGCG 160076. The optical center of the galaxy is indicated with a cross. The numbers indicate heliocentric velocity in $\mathrm{kms}^{-1}$. The $F W H M$ is indicated by the circle, $29.9^{\prime \prime} \times 26.9^{\prime \prime}$.

\section{KUG 1258+287, FOCA 195 and CGCG 160-098}

These objects are part of a group dominated by CGCG 160-098 (see Biviano et al. 1996). As all the galaxies in Coma detected in Hi outside the X-ray emission, this group shows a normal Hi content and a regular gas distribution (Figs. 11a, 12a and 13a), with the Hi more

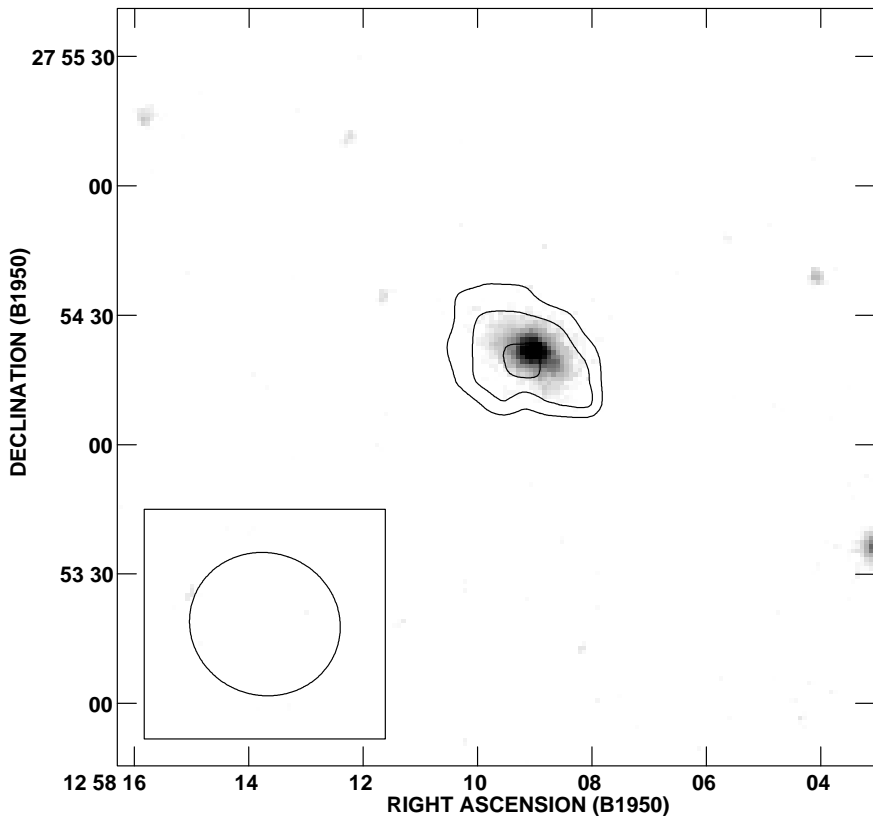

Fig. 8. Hi density distribution of CGCG 160-086, superposed on a DSS $B$-band gray scale image. The contours are 0.4 $(2.5 \sigma), 0.9$, and $1.3 \times 10^{20} \mathrm{~cm}^{-2}$. The $F W H M$ is indicated by the circle, $35.2^{\prime \prime} \times 33.0^{\prime \prime}$.

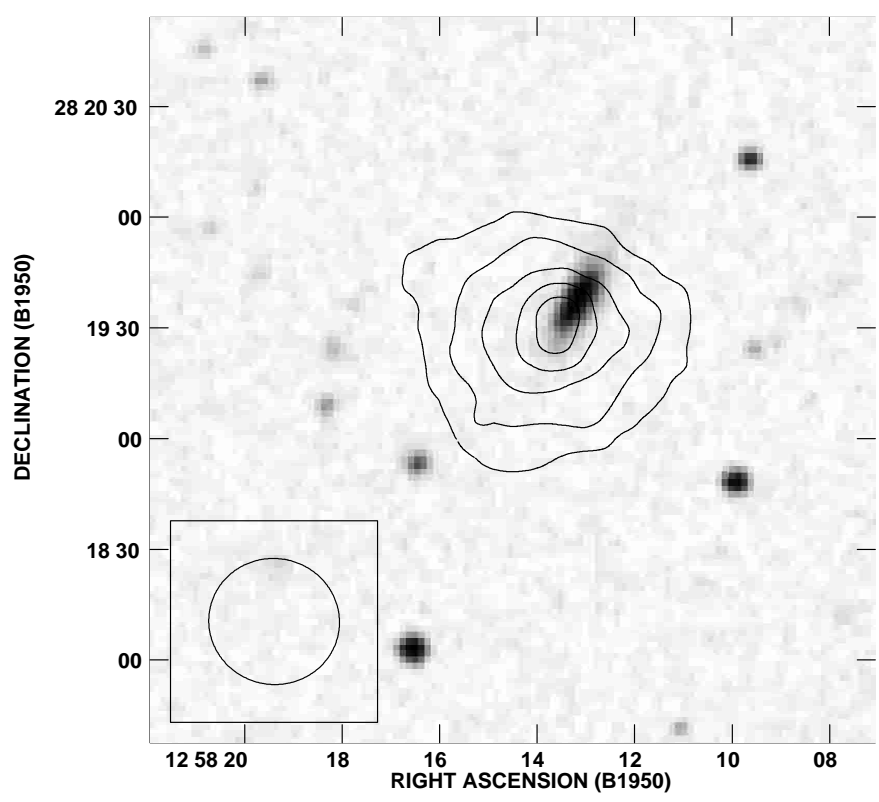

Fig. 9a. Hi density distribution of IC 4040, superposed on a DSS $B$-band gray scale image. The contours are $0.2(5 \sigma), 0.4$, $0.7,1.1$, and $1.4 \times 10^{20} \mathrm{~cm}^{-2}$. The FWHM is indicated by the circle, $35.5^{\prime \prime} \times 34.1^{\prime \prime}$.

extended than the optical disk and coinciding with the optical position. Particular features of these galaxies are their systematic blue $m_{\mathrm{UV}}-b$ color (see Table 2 and Fig. 7 in Paper I), and their high radial velocity (between $8400 \mathrm{~km} \mathrm{~s}^{-1}$ and $8880 \mathrm{~km} \mathrm{~s}^{-1}$ ), indicating a fast accretion of the group towards the main cluster body. Normal rotation patterns are shown by the bluest objects KUG 1258+287 and FOCA 195 (see Figs. 11b and 12b). 


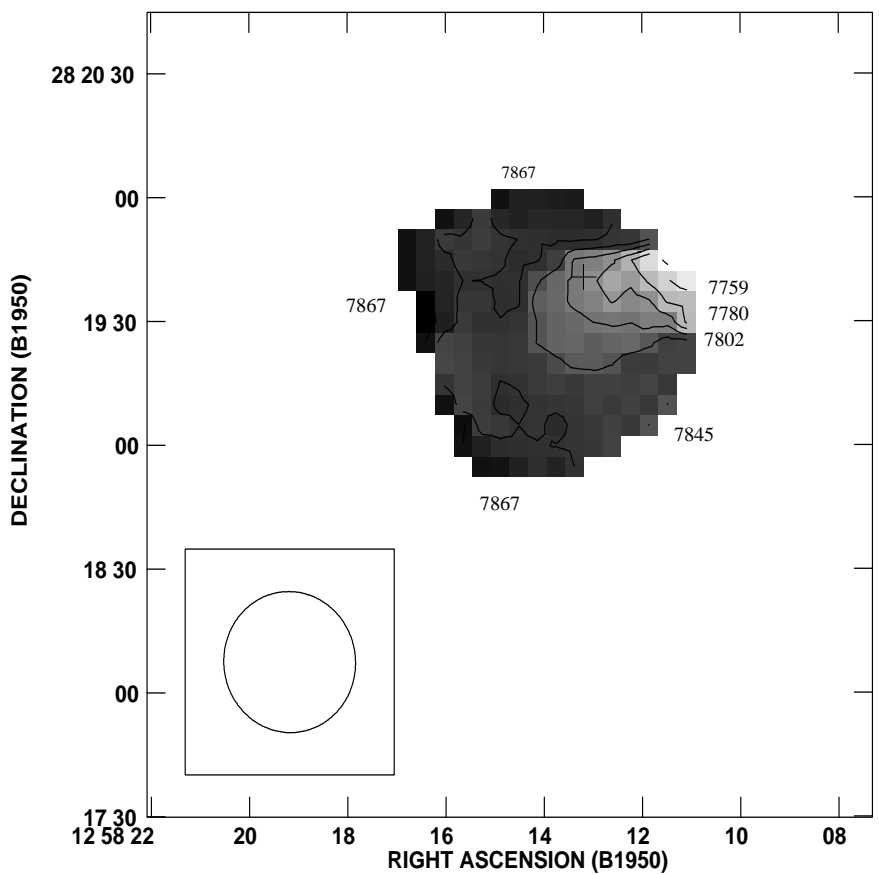

Fig. 9b. Intensity weighted mean velocity field of IC 4040. The optical center of the galaxy is indicated with a cross. The numbers indicate heliocentric velocity in $\mathrm{km} \mathrm{s}^{-1}$. The $F W H M$ is indicated by the circle, $35.5^{\prime \prime} \times 34.1^{\prime \prime}$.

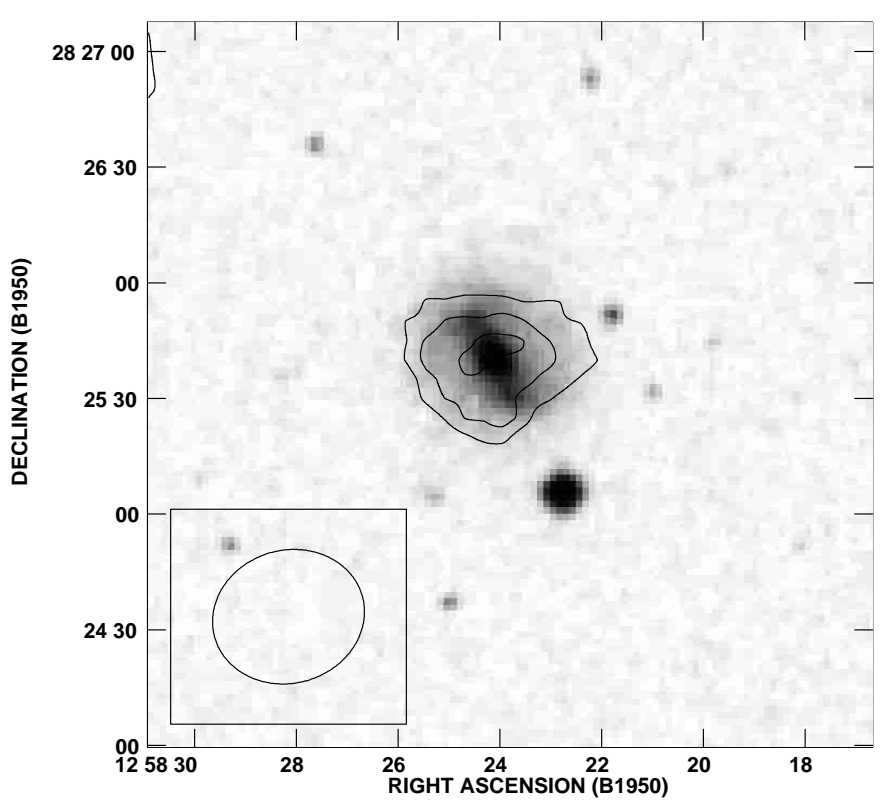

Fig. 10. Hi density distribution of NGC 4907, superposed on a DSS $B$-band gray scale image. The contours are $0.2(2.5 \sigma)$, 0.5 , and $0.9 \times 10^{20} \mathrm{~cm}^{-2}$. The $F W H M$ is indicated by the circle, $39.8^{\prime \prime} \times 34.5^{\prime \prime}$.

HI emission equivalent to $2.2 \times 10^{8} M_{\odot}$ was detected at $1.4^{\prime} \mathrm{W}$ of KUG $1258+287$ but no optical counterpart is observed.

\section{NGC 4911}

This is one of the two brightest spirals in Coma. The Hi morphology of NGC 4911 shows a shrunken disk with two central peaks and a normal surface density in

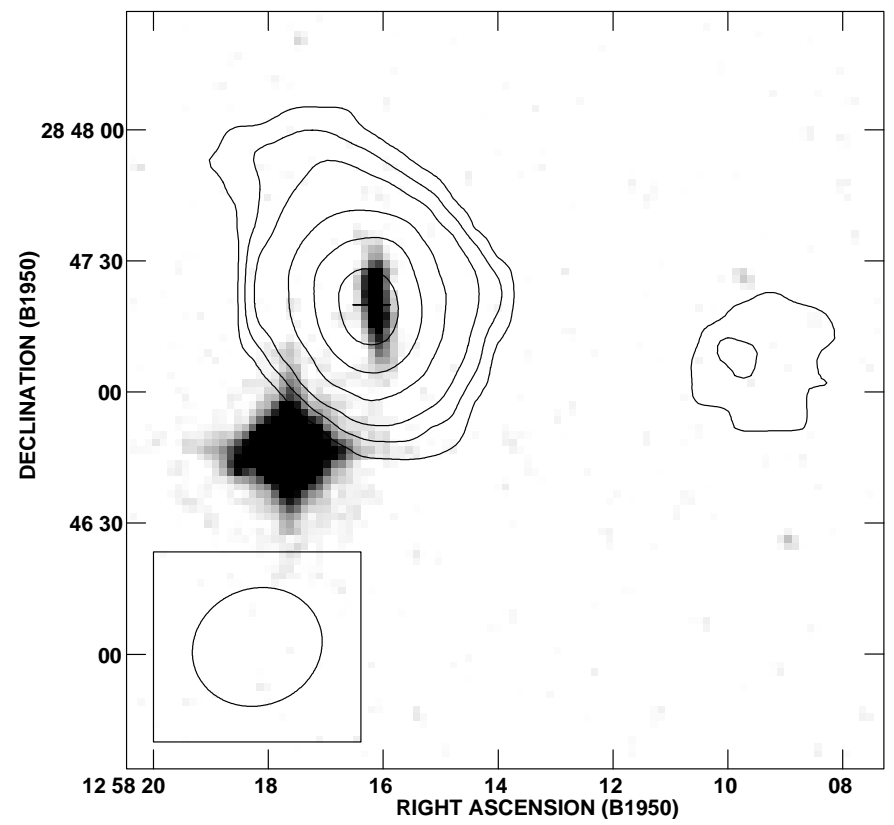

Fig. 11a. Hi density distribution of KUG $1258+287$, superposed on a DSS $B$-band gray scale image. The contours are $0.3(2.5 \sigma), 1.1,2.2,4.5,6.8$, and $9.0 \times 10^{20} \mathrm{~cm}^{-2}$. The $F W H M$ is indicated by the circle, $30.2^{\prime \prime} \times 26.7^{\prime \prime}$.

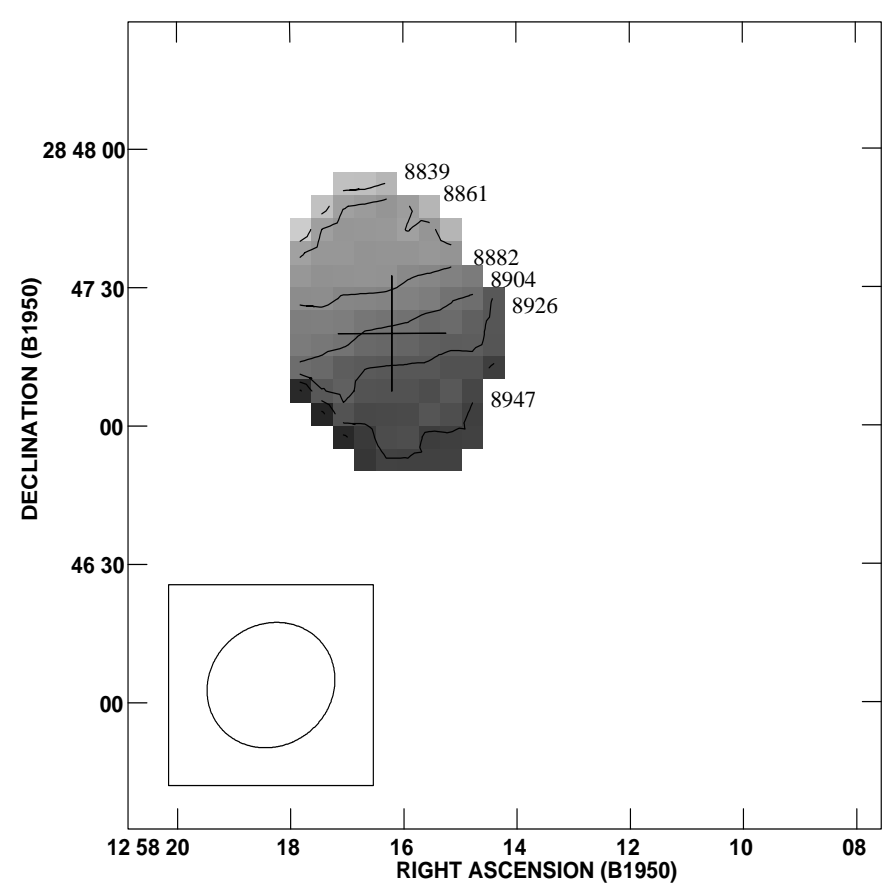

Fig. 11b. Intensity weighted mean velocity field of KUG $1258+287$. The optical center of the galaxy is indicated with a cross. The numbers indicate heliocentric velocity in $\mathrm{kms}^{-1}$. The $F W H M$ is indicated by the circle, $30.2^{\prime \prime} \times 26.7^{\prime \prime}$.

the central region as predicted by ram-pressure stripping (Fig. 14a). A shift in position is shown between the HI and the optical, the former lying about $4 \mathrm{kpc}$ south of the optical component. NGC 4911 shows a very high total gas content, $M_{\mathrm{HI}}+M_{\mathrm{H} 2} \sim 3.7 \times 10^{9} M_{\odot}$ (Casoli et al. 1996), 


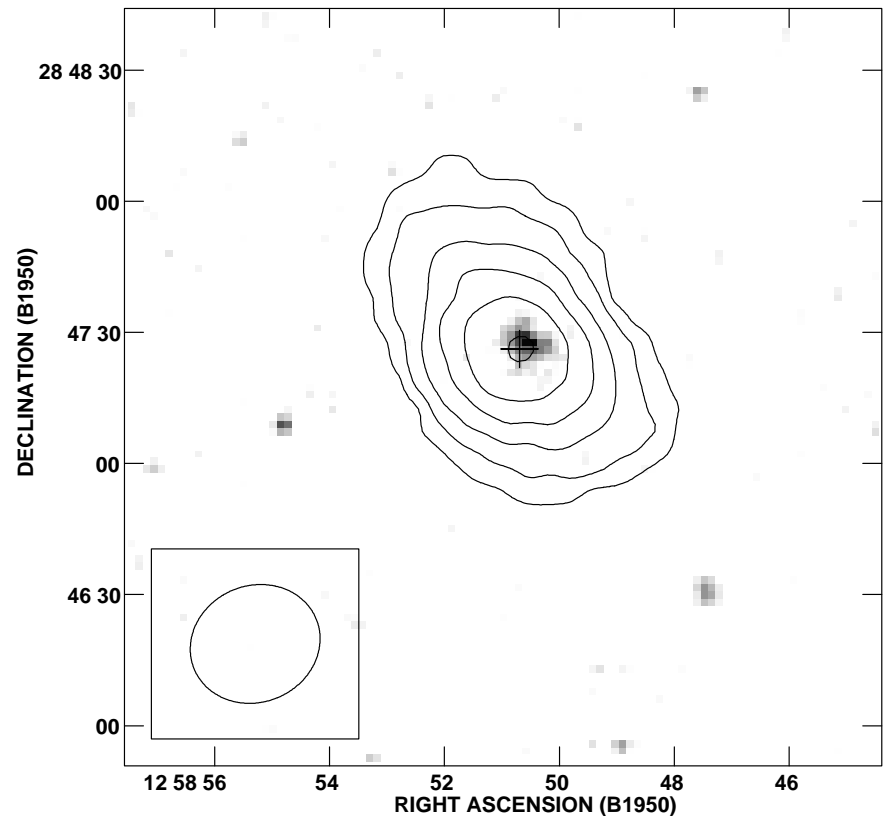

Fig. 12a. HI density distribution of FOCA 0195, superposed on a DSS $B$-band gray scale image. The contours are 0.2 $(2.5 \sigma), 0.9,1.8,2.6,3.5$, and $4.4 \times 10^{20} \mathrm{~cm}^{-2}$. The $F W H M$ is indicated by the circle, $30.2^{\prime \prime} \times 26.7^{\prime \prime}$.

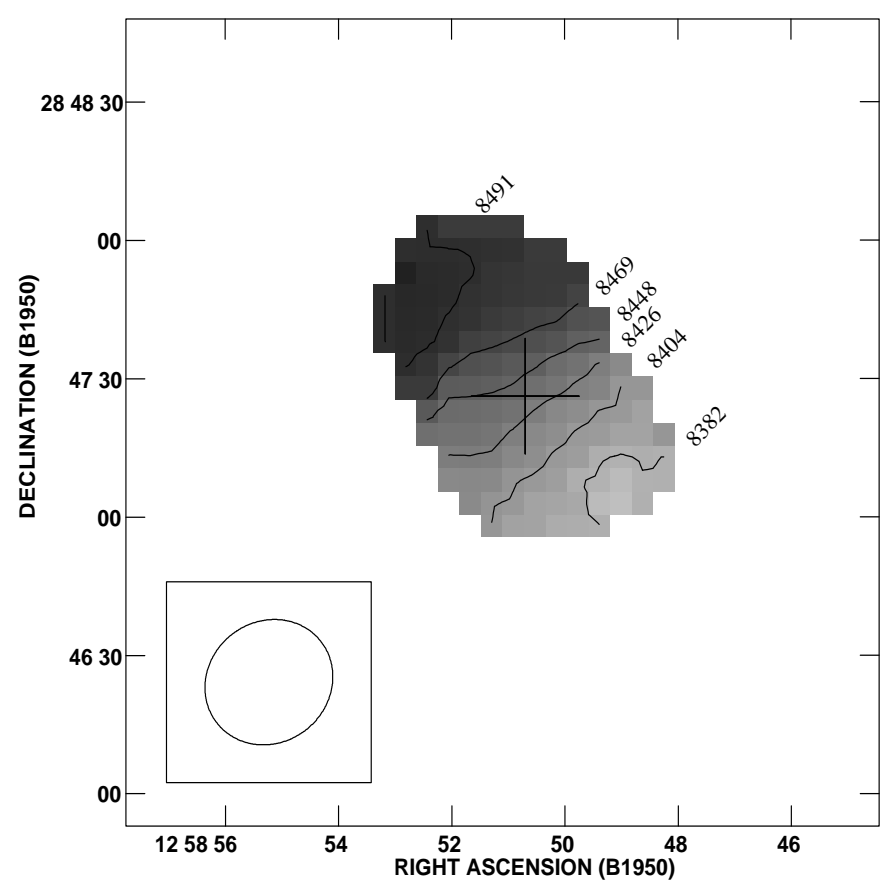

Fig. 12b. Intensity weighted mean velocity field of FOCA 0195. The optical center of the galaxy is indicated with a cross. The numbers indicate heliocentric velocity in $\mathrm{km} \mathrm{s}^{-1}$. The $F W H M$ is indicated by the circle, $30.2^{\prime \prime} \times 26.7^{\prime \prime}$.

but only a small fraction $\left(M_{\mathrm{HI}} / M_{\mathrm{H} 2}=0.34\right)$ is in atomic form. Considering the $\mathrm{CO}$ observations by Lavezzi et al. (1999) this fraction is much lower: $M_{\mathrm{HI}} / M_{\mathrm{H} 2}=0.22$. The velocity field shows normal rotation of the inner disk (see Fig. 14b) along an axis oriented roughly SE-NW.

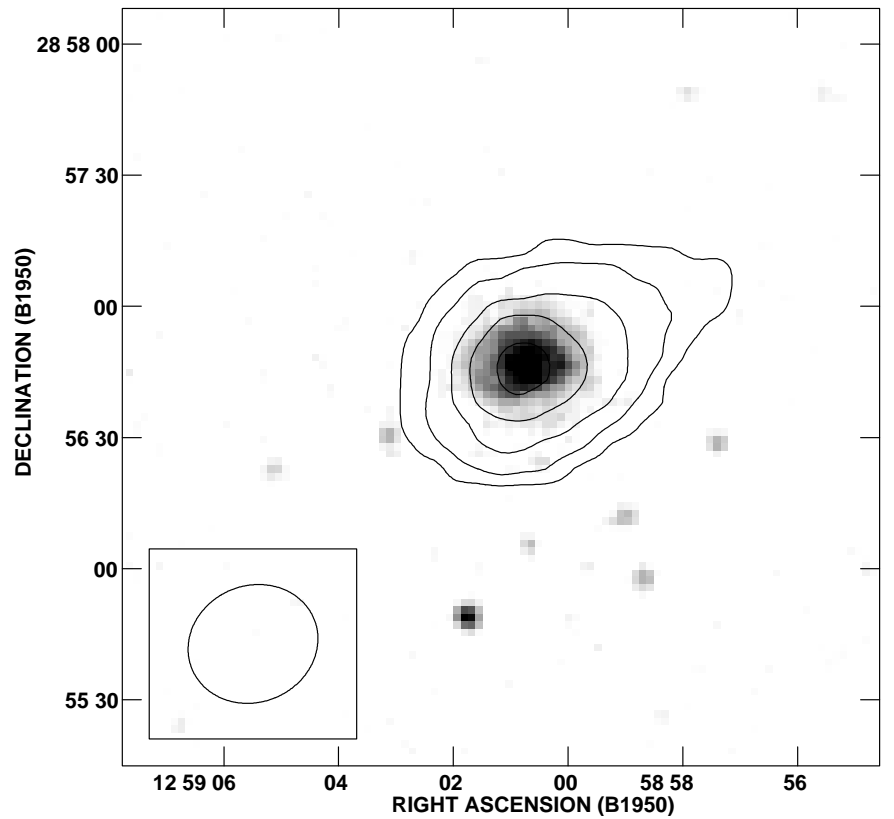

Fig. 13a. Hi density distribution of CGCG 160-098, superposed on a DSS $B$-band gray scale image. The contours are $0.3(2.5 \sigma), 1.1,2.2$, and $4.4 \times 10^{20} \mathrm{~cm}^{-2}$. The $F W H M$ is indicated by the circle, $30.2^{\prime \prime} \times 26.7^{\prime \prime}$.

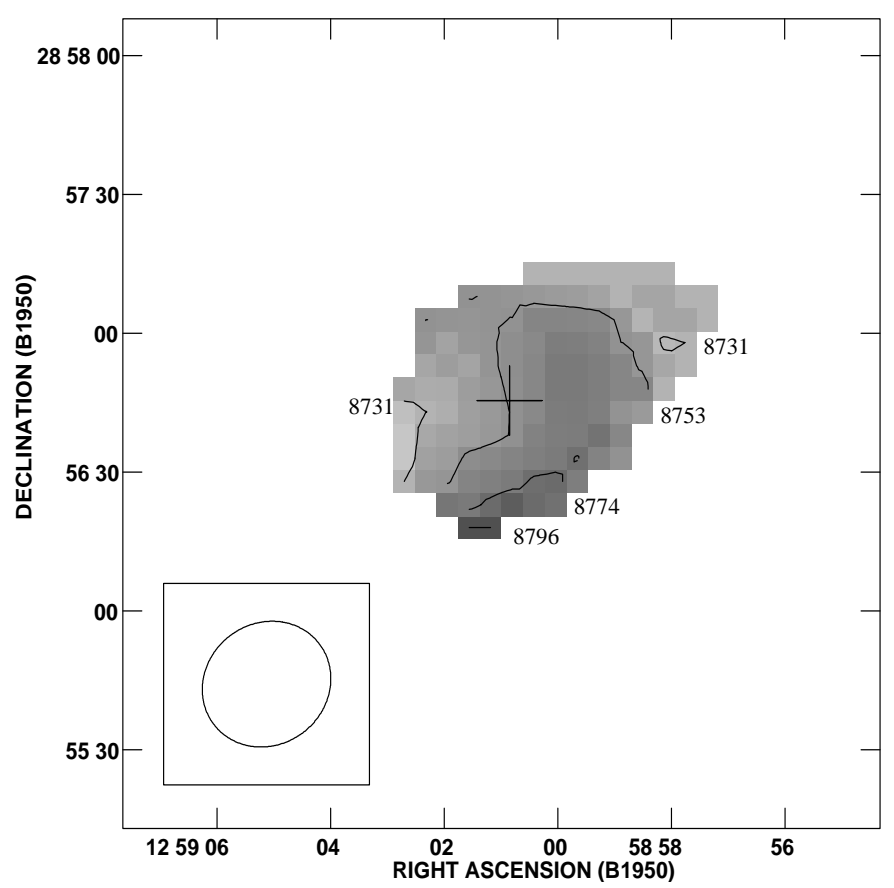

Fig. 13b. Intensity weighted mean velocity field of CGCG 160098. The optical center of the galaxy is indicated with a cross. The numbers indicate heliocentric velocity in $\mathrm{kms}^{-1}$. The $F W H M$ is indicated by the circle, $30.2^{\prime \prime} \times 26.7^{\prime \prime}$.

NGC 4911, projected $20^{\prime} \mathrm{SE}$ of NGC $4874\left(v_{\text {rel }} \sim\right.$ $1000 \mathrm{~km} \mathrm{~s}^{-1}$ ), is thought to be the dominant galaxy of a group that recently crossed the cluster core (Biviano et al. 1996). Vikhlinin et al. (1997) reported an X-ray filamentary structure in this region, crossing over NGC 4911 and culminating at the position of NGC 4921. This cool spot 


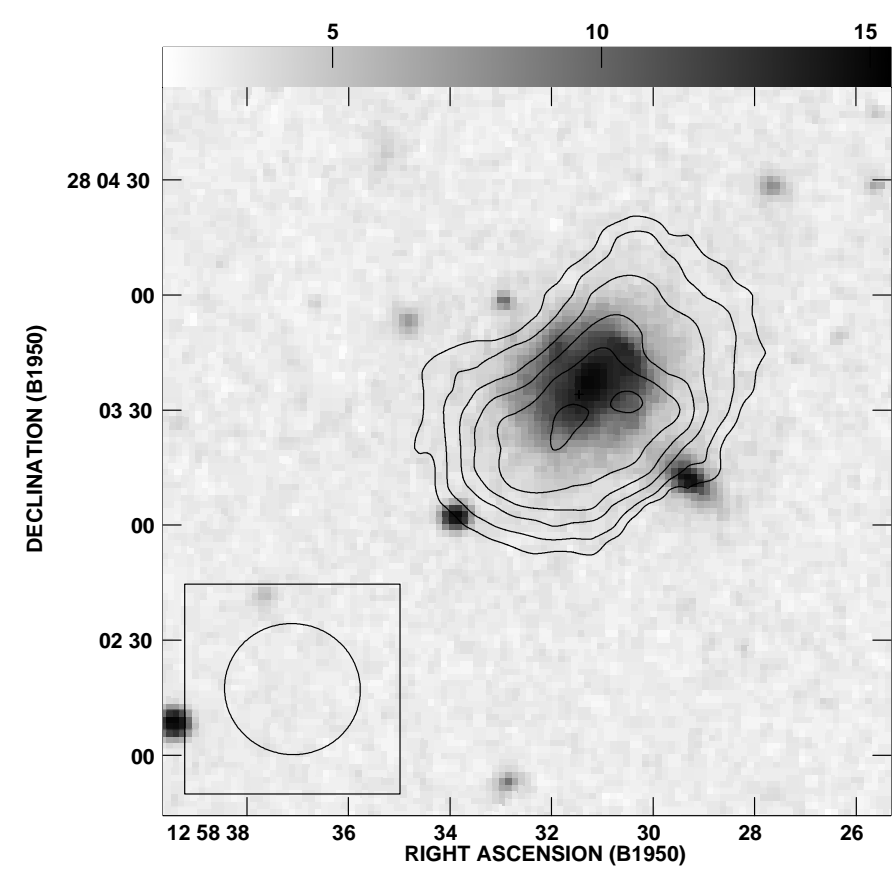

Fig. 14a. Hi density distribution of NGC 4911, superposed on a DSS $B$-band gray scale image. The contours are $0.3(2.5 \sigma)$, 1.4 , and $2.0 \times 10^{20} \mathrm{~cm}^{-2}$. The $F W H M$ is indicated by the circle, $29.8^{\prime \prime} \times 27.7^{\prime \prime}$.

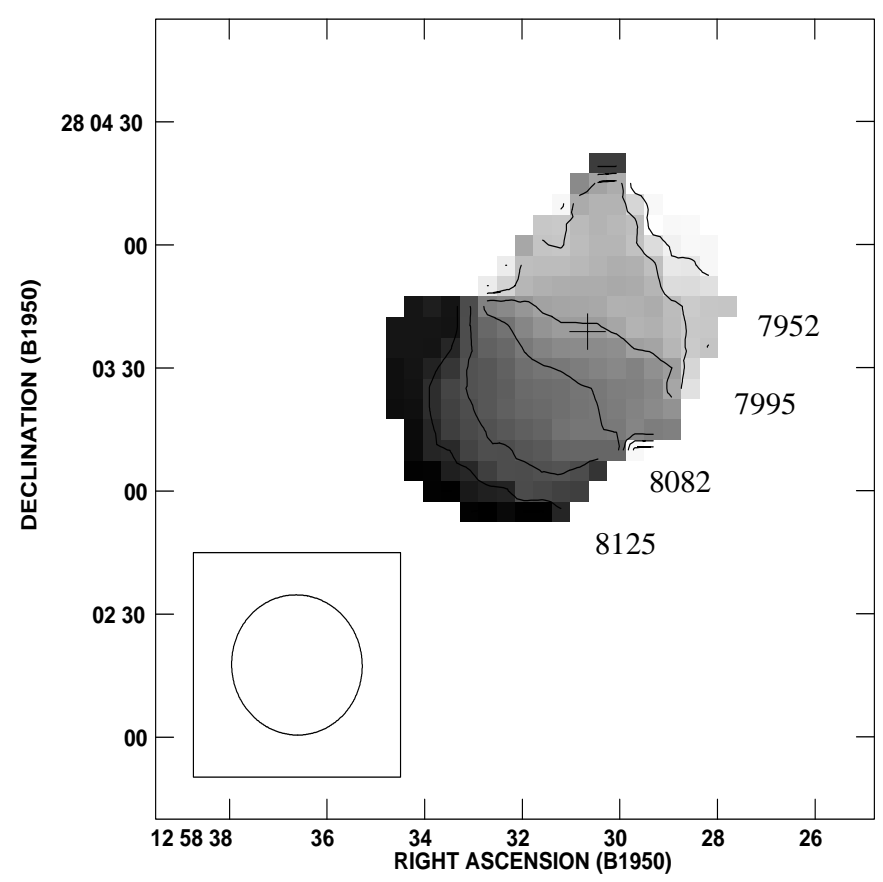

Fig. 14b. Intensity weighted mean velocity field of NGC 4911. The optical center of the galaxy is indicated with a cross. The numbers indicate heliocentric velocity in $\mathrm{km} \mathrm{s}^{-1}$. The $F W H M$ is indicated by the circle, $29.8^{\prime \prime} \times 27.7^{\prime \prime}$.

has recently been confirmed with XMM (Arnaud et al. 2001), who consider the possibility of gas stripped from an infalling group, but not directly produced in the Coma center. They found that part of the X-ray excess in this zone is due to NGC 4911. No other galaxies of this group

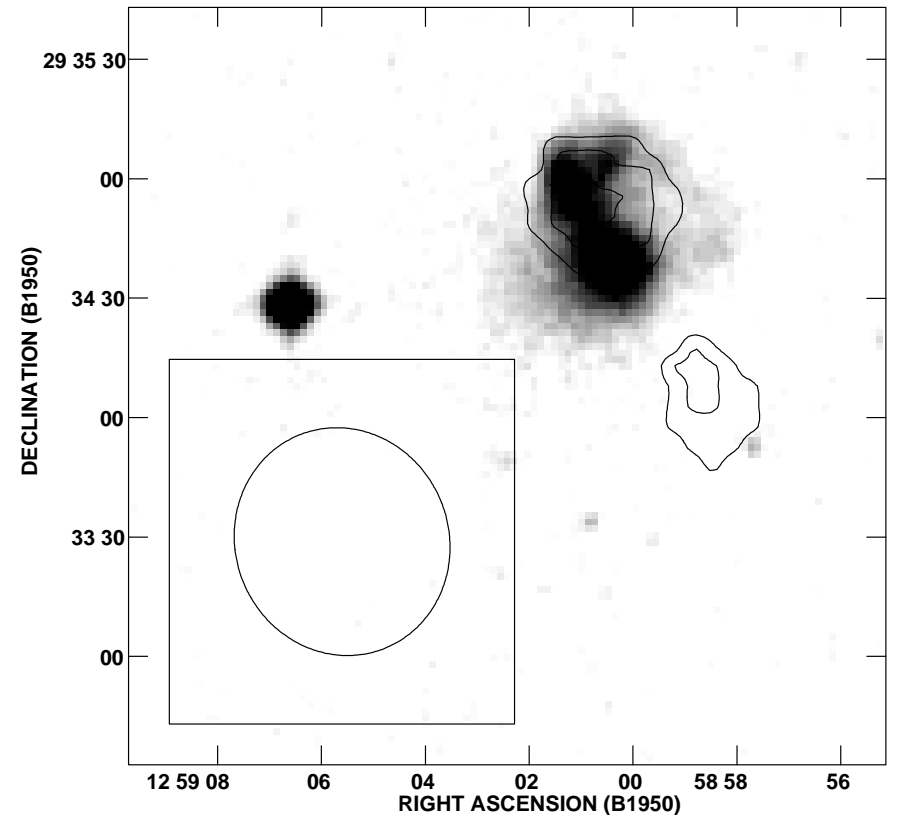

Fig. 15. Hi density distribution showing the emission feature of NGC 4922, superposed on a DSS B-band gray scale image. Strong Hi absorption is present which attenuates the emission. The contours are $0.2(2.5 \sigma), 0.4$, and $0.5 \times 10^{20} \mathrm{~cm}^{-2}$. The $F W H M$ is indicated by the circle, $57.8^{\prime \prime} \times 53.4^{\prime \prime}$.

were detected in Hi, in support of the stripping hypothesis. However, the fact that none of the 14 catalogued galaxies (Biviano et al. 1996) have been classified as spirals weakens this argument somewhat.

\section{NGC 4922}

This is the best known case of a merger in Coma, and the only pair detected in this survey; it is located in the northern most field, $1.4^{\circ}$ from the cluster center. It is very bright in radio $(27.5 \mathrm{mJy})$ and the brightest IR source in Coma (Mirabel \& Sanders 1988). NGC 4922 consists of two merging galaxies, one spiral in the north and one early type galaxy in the south. We detected Hi in emission and absorption. The Hi shown in Fig. 15, centered in the spiral galaxy, is strongly attenuated by the absorption. An $\mathrm{HI}$ emission equivalent to $5.8 \times 10^{7} M_{\odot}$ was detected south of the merging system, but no optical counterpart is evident from the DSS image.

\section{NGC 4921}

This is the brightest spiral in Coma, located $24^{\prime}(\sim 0.5 \mathrm{Mpc})$ SE from NGC 4874. It roughly coincides with a second order X-ray peak (Vikhlinin et al. 1997) also detected with XMM by Arnaud et al. (2001) and Briel et al. (2001). NGC 4921 presents a very peculiar picture in $21 \mathrm{~cm}$ : a double peaked Hi disk (Fig. 16a) which is considerably smaller than the optical one, with a strongly asymmetric distribution, with most of the neutral gas distributed along the SE spiral arm. The gaseous disk presents a striking shift in position of $\sim 25^{\prime \prime}$ (some $8 \mathrm{kpc}$ ) SE from the optical. The velocity field is shown in Fig. $16 \mathrm{~b}$. 


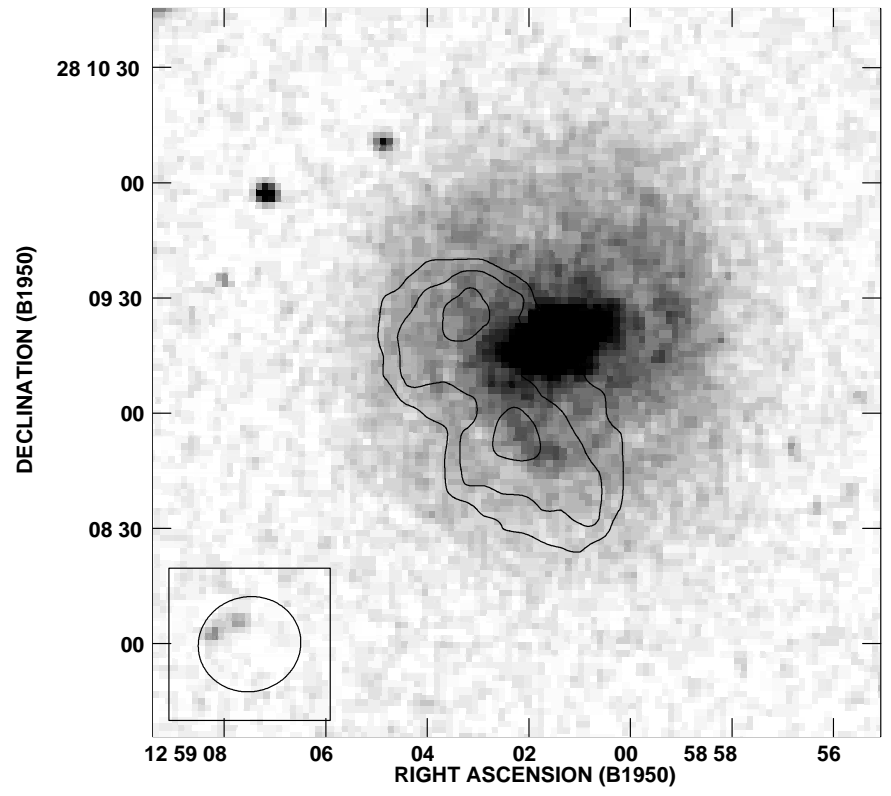

Fig. 16a. Hi density distribution of NGC 4921, superposed on a DSS $B$-band gray scale image. The contours are $0.3(2.5 \sigma)$, $1.2,1.7$, and $2.3 \times 10^{20} \mathrm{~cm}^{-2}$. The $F W H M$ is indicated by the circle, $39.8^{\prime \prime} \times 34.5^{\prime \prime}$.

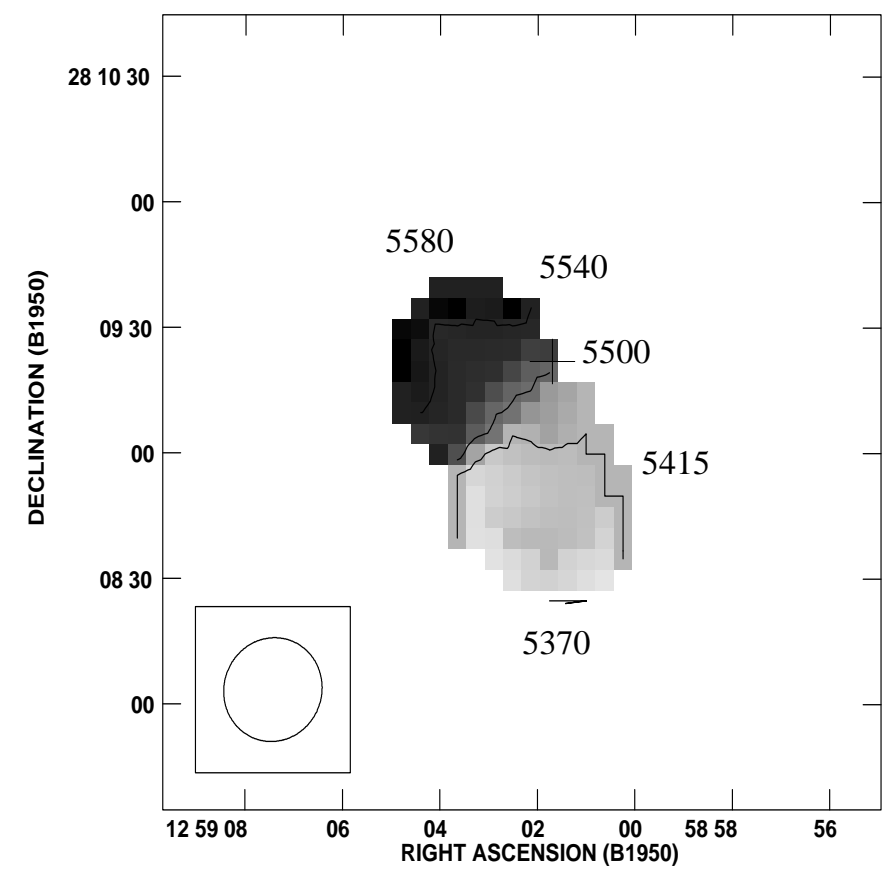

Fig. 16b. Intensity weighted mean velocity field of NGC 4921. The optical center of the galaxy is indicated with a cross. The numbers indicate heliocentric velocity in $\mathrm{km} \mathrm{s}^{-1}$. The $F W H M$ is indicated by the circle, $39.8^{\prime \prime} \times 34.5^{\prime \prime}$.

NGC 4921 is perhaps the best example in Coma where several mechanisms are present simultaneously. (a) The shrunken Hi disk and the shift between optical and Hi positions look like clear signatures of ram-pressure stripping, which is also supported by the supersonic velocity of the galaxy relative to the cluster, $1521 \mathrm{~km} \mathrm{~s}^{-1}$. (b) NGC 4921 shows the largest cross section in our sample, which could

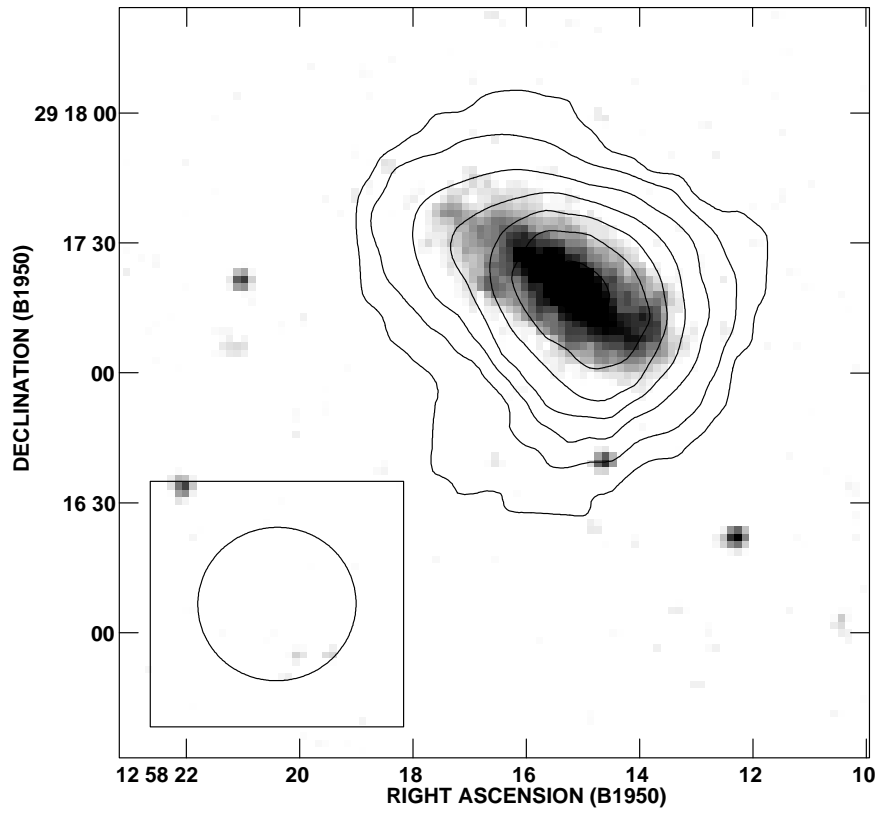

Fig. 17a. Hi density distribution of IC 842 superposed on a DSS $B$-band gray scale image. The contours are $0.3(2.5 \sigma)$, $1.2,2.5,3.7,4.9,6.2$, and $7.4 \times 10^{20} \mathrm{~cm}^{-2}$. The $F W H M$ is indicated by the circle, $36.6^{\prime \prime} \times 35.4^{\prime \prime}$.

produce, by viscous stripping, a mass loss rate value up to $20 M_{\odot} \mathrm{yr}^{-1}$. Another feature supporting viscous stripping is the relatively low surface gas density in the central region of NGC 4921, previously classified as anemic by van den Bergh (1976). (c) The NW zone, where the brightest HII regions are seen (Amram et al. 1992), appears depleted of HI, similar to the case of NGC 4848. Furthermore, NGC 4921 displays a high total gas content, $M_{\mathrm{HI}}+M_{\mathrm{H} 2}=2.65 \times 10^{9} M_{\odot}$, with only a small fraction (0.36) in atomic form, suggesting that $\mathrm{HI}$ is actively converted to molecular gas. Gas re-accretion in the NW may also be present, triggering the HII regions along the spiral arm. As this galaxy does not show any optical distortion, processes involving gravitational effects are unlikely to be important.

\section{IC 842 and IC 4088}

These galaxies lie in the far northern region of Coma, along the supercluster NE filament, and far from the $\mathrm{X}$-ray emission. As expected, they display normal Hi content and no effects of interaction with the ICM are seen (Figs. 17a and 18a). They also display normal rotation patterns (Figs. 17b and 18b). Three dwarf systems were detected in Hi around IC 4088, one of them ([GMP 83] $1866)$ lies at only $2^{\prime}(\sim 40 \mathrm{kpc})$ north of the spiral, but no optical distortions are evident. The dwarfs show velocity dispersions between $43 \mathrm{kms}^{-1}$ and $173 \mathrm{kms}^{-1}$, and their HI masses range between 0.2 and $2.0 \times 10^{9} M_{\odot}$. All the galaxies detected in this region, including the merger NGC 4922, are likely part of a group falling towards to the cluster center (Paper I). 


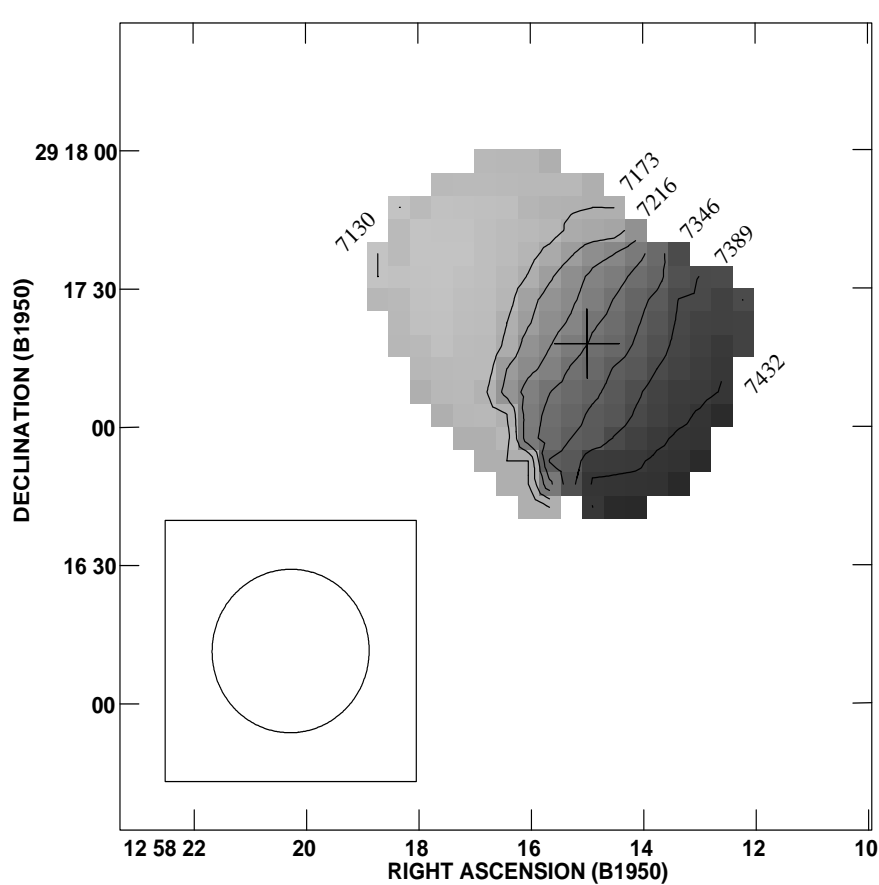

Fig. 17b. Intensity weighted mean velocity field of IC 842 . The optical center of the galaxy is indicated with a cross. The numbers indicate heliocentric velocity in $\mathrm{km} \mathrm{s}^{-1}$. The FWHM is indicated by the circle, $36.6^{\prime \prime} \times 35.4^{\prime \prime}$.

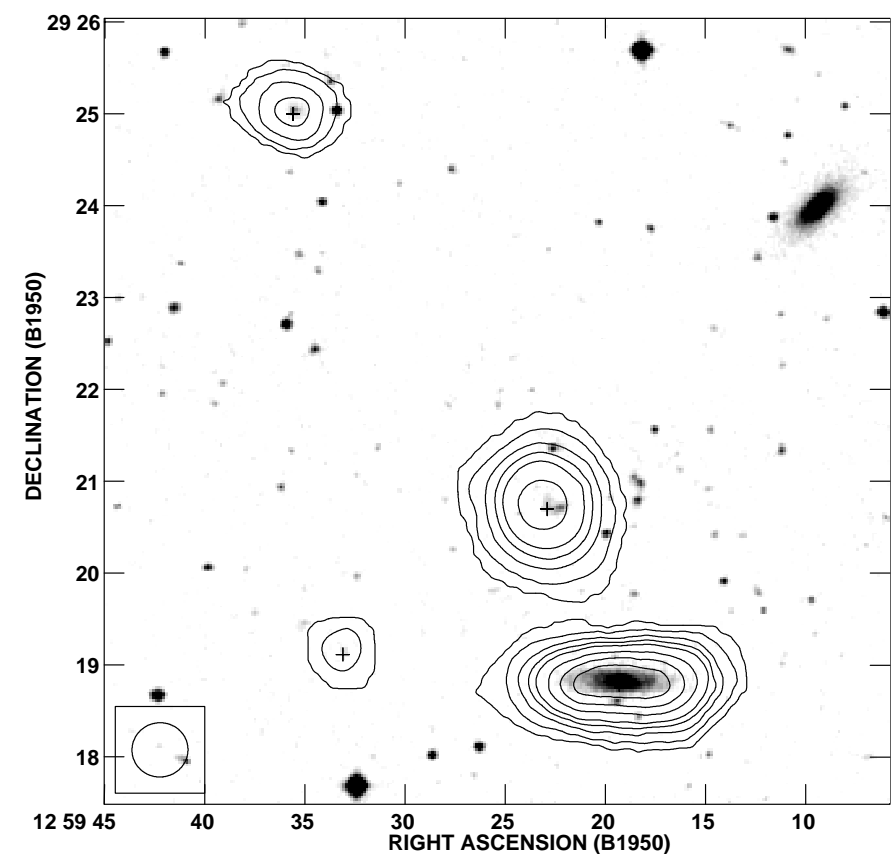

Fig. 18a. Hi density distribution of IC 4088 and 3 neighbor dwarf systems, superposed on a DSS $B$-band gray scale image. The contours are $0.2(2.5 \sigma), 1.0,1.9,2.9,3.9,5.8,7.8$, and $9.7 \times$ $10^{20} \mathrm{~cm}^{-2}$. The FWHM is indicated by the circle, $36.6^{\prime \prime} \times 35.4^{\prime \prime}$.

\section{CGCG 160-106}

This blue disk galaxy is located in the SE outskirts of the $\mathrm{X}$-ray emission. The ICM does not exert important effects on this galaxy because of the low ICM density at the galaxy position, the low value of $v_{\text {rel }}$, and the small

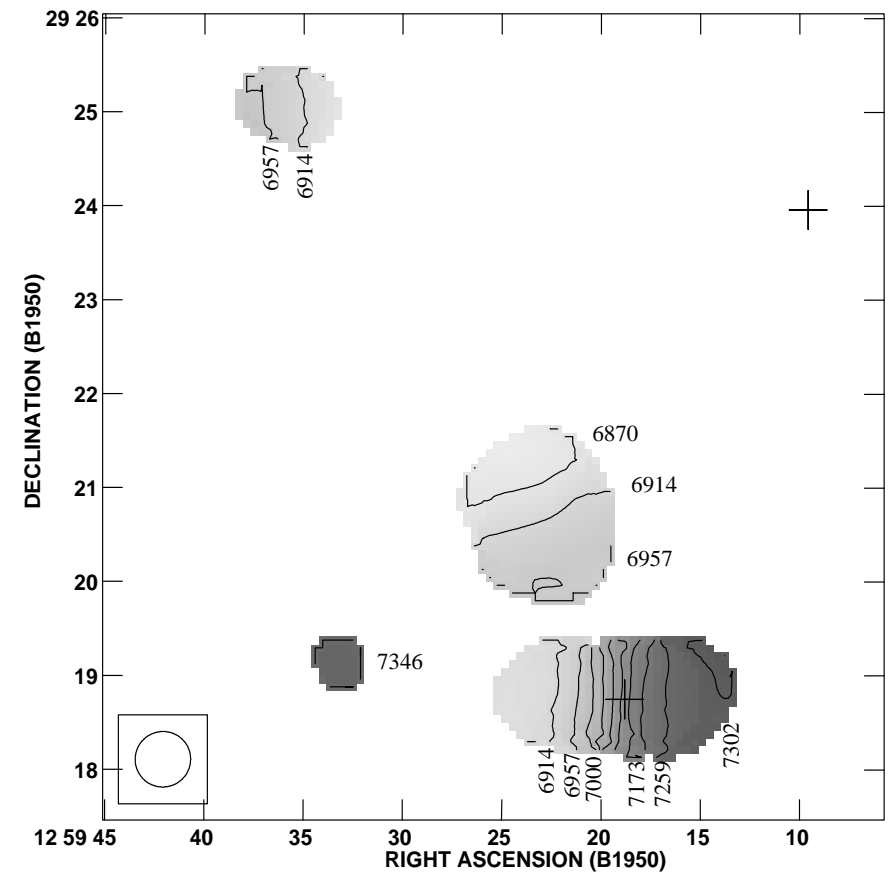

Fig. 18b. Intensity weighted mean velocity field of IC 4088 . The optical center of the galaxies are indicated with crosses. The numbers indicate heliocentric velocity in $\mathrm{kms}^{-1}$. The $F W H M$ is indicated by the circle, $36.6^{\prime \prime} \times 35.4^{\prime \prime}$.

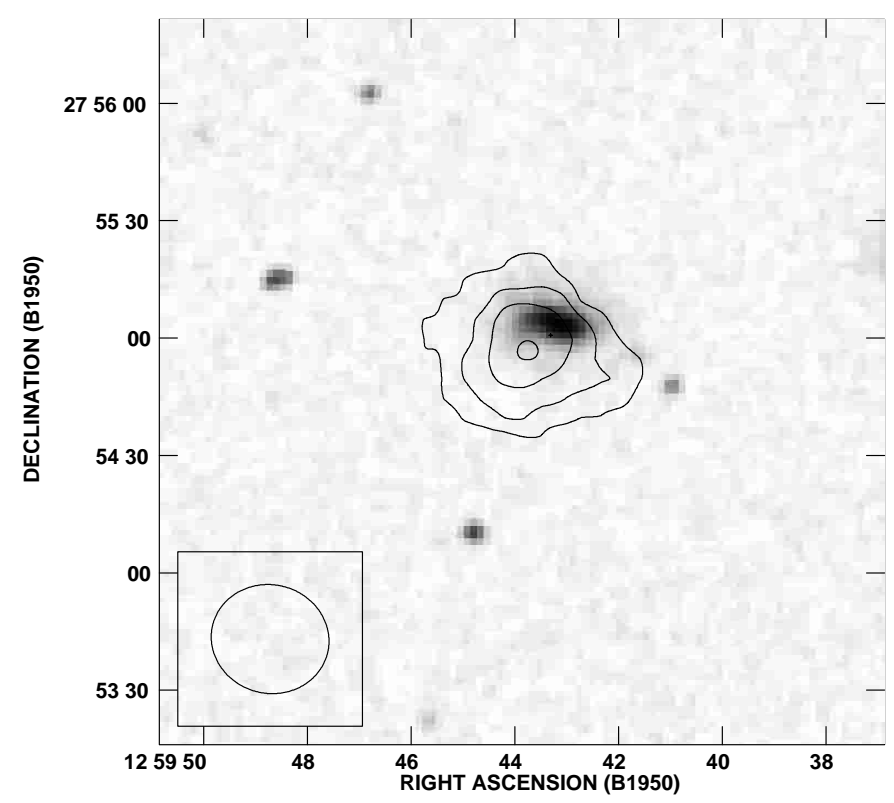

Fig. 19. Hi density distribution of NGC 4926-A, superposed on a DSS $B$-band gray scale image. The contours are 0.3 $(2.5 \sigma), 1.4$, and $2.1 \times 10^{20} \mathrm{~cm}^{-2}$. The $F W H M$ is indicated by the circle, $35.2^{\prime \prime} \times 33.0^{\prime \prime}$.

cross section. CGCG 160-106 is not very HI poor (it is deficient by a factor of 1.5), and the Hi morphology, barely resolved in Fig. 19, only shows a shrunken disk slightly bigger than the optical one.

We detect a clear shift in the $\mathrm{HI}$ position, lying $15^{\prime \prime}$ (some $5 \mathrm{kpc}$ ) SW from the optical disk, and an intriguing difference in velocity between the $\mathrm{HI}$ and 
optical: $6876 \mathrm{kms}^{-1}$ and $7188 \mathrm{kms}^{-1}$, respectively. Amram et al. (1992) observed this galaxy in $\mathrm{H} \alpha$ and reported a velocity of $7100 \mathrm{~km} \mathrm{~s}^{-1}$ and an extension to the SW, coincident with the position of a dwarf companion separated by $\sim 20^{\prime \prime}$. We detect weak Hi emission in the same zone, but higher resolution is needed to resolve the dwarf system.

\section{The $\mathrm{HI}$ and the ICM in Coma}

As mentioned earlier, physical mechanisms affecting galaxies in clusters are produced by interactions with one or more of the next three elements: the hot ICM, neighbor galaxies, and the cluster gravitational field. Two effects observed in Hi in this work are explained on the basis of ICM-ISM interactions: the position of HI deficient galaxies relative to the ICM as drawn by the $\mathrm{X}$-ray emission, and the fact that the most central galaxies detected in HI appear stripped in their outer regions, as predicted for ram-pressure stripping. If tidal interactions were predominant there should be evidence of optical signatures of the interaction, but none of the $\mathrm{HI}$ deficient galaxies in this work (except the merger NGC 4922), neither detected nor undetected, display peculiarities in their optical morphology. It was also shown in Paper I that tidal interactions are unlikely to be the explanation for the disturbed Hi disks in Coma or the triggering of starburst events, because there is no correlation between these effects and the number of close neighbors.

In order to confirm the role of ram pressure stripping in producing the observed Hi distributions and in enhancing the star formation in Coma, it is fundamental to compare the HI imaging with 3D simulations of the ISM-ICM interaction. A thorough comparison will help to estimate the role played by different infalling orbits, inclination angles, and ICM densities, as well as to determine the stripping time scale and the regions of the disk where ram-pressure is more effective. To this aim we compare our HI observations with predictions made by $3 \mathrm{D}$-simulations carried out by Abadi et al. (1999) and Vollmer et al. (2001b).

Simulations by Abadi et al. (1999) are a good point of departure even if the clumpiness of the ISM and different elapsed times after crossing the cluster core are not taken into account. They computed the expected radius of a ram pressure stripped gas disk $\left(R_{\text {str }}\right)$ for a Coma clusterlike density and dispersion velocity. For instance, a typical galaxy in Coma with $v_{\text {rel }} \sim 1000 \mathrm{~km} \mathrm{~s}^{-1}$ would have $R_{\text {str }} \sim 6$ kpc. In Table 1 we compare the observed Hi radius (or upper limits if resolution is marginal) with the prediction of $R_{\text {str }}$ made by Abadi et al. (1999). Columns 1 and 2 give the galaxy identification, Col. 3 gives the radial velocity relative to the cluster. In Col. 4 we give the observed HI radius in kpc estimated at a level of $3 \times 10^{19} M_{\odot}$, taking the average between major and minor Hi axis (none of the galaxies in Table 1 are very elongated). Column 5 gives the observed $\mathrm{HI}$ radii corrected for the beam; for galaxies marginally resolved (indicated with $*$ ) we give 0.5 times the beam size as an upper limit of the beam corrections,
Table 1. Comparison between the observed and predicted Hi radius in $\mathrm{kpc}$, for central galaxies in Coma.

\begin{tabular}{clrrcr}
\hline CGCG & Other & $\begin{array}{r}v_{\text {rel }} \\
\mathrm{km} \mathrm{s}^{-1}\end{array}$ & $\begin{array}{r}\text { Obs } \\
r_{\mathrm{HI}}\end{array}$ & $\begin{array}{l}\text { Corr } \\
r_{\mathrm{HI}}\end{array}$ & $\begin{array}{r}\text { Pred } \\
r_{\mathrm{HI}} \\
(1)\end{array}$ \\
name & $(3)$ & $(4)$ & $(5)$ & $(6)$ \\
\hline $160-055$ & NGC 4848 & 41 & 6.8 & $5.0^{*}$ & 17.0 \\
$160-073$ & Mrk 058 & 1575 & 7.9 & 5.8 & 4.5 \\
$160-086$ & & 481 & 6.0 & $5.6^{*}$ & 8.5 \\
$160-252$ & IC 4040 & 758 & 10.2 & 8.0 & 7.5 \\
$160-257$ & NGC 4907 & 1180 & 6.6 & $5.8^{*}$ & 5.5 \\
$160-260$ & NGC 4911 & 997 & 12.7 & 12.2 & 6.5 \\
$160-095$ & NGC 4921 & 1521 & 11.2 & 10.2 & 5.0 \\
$160-106$ & NGC 4926-A & 124 & 8.2 & 5.7 & 14.0 \\
\hline
\end{tabular}

(*) Galaxies marginally resolved in Hı.

following Wild (1970). Column 6 gives the predicted value of $R_{\mathrm{str}}$ from Abadi et al. (1999), considering the corresponding velocity of the galaxy relative to the cluster.

The predicted Hi radius is calculated assuming that the galaxy's distance to the center equals its projected distance and its velocity through the cluster equals its radial velocity. Considering those assumptions there is good agreement between observed and predicted values of the $\mathrm{HI}$ radius. The two galaxies (NGC 4848 and 4926-A) that have significantly smaller radii than predicted must have a non negligible velocity in the plane of the sky, while the giants NGC 4911 and 4921 are probably at larger distance from the center and only in projection very close. For the three unresolved galaxies the HI distribution is limited to a central region of $\leq 6 \mathrm{kpc}$. Our results for $\mathrm{HI}$ deficient yet detected galaxies in Coma, confirm that most of the restoring force is coming from the central parts of the disk where the presence of the bulge is more important, as found by Abadi et al.'s simulation. They found that the gas is not completely removed by ram pressure, suggesting that other processes may be at work affecting those spirals which are not detected in HI in this survey.

Three dimensional simulations taking into account the clumpiness of the ISM were carried out for the Virgo cluster by Vollmer et al. (2001b). These authors found that stripping is very effective for galaxies that are on radial orbits through the cluster, in agreement with observational evidence provided by Dressler (1984) and Solanes et al. (2000). Vollmer et al. (2001b) found that time scales for ram pressure effects in Virgo may be as short as $3 \times 10^{7} \mathrm{yr}$, and in Coma they would likely be shorter because of its larger core size and more hostile ICM conditions. Interestingly, those authors found that galaxies showing important gas disruptions are not infalling but have already gone through the cluster core. If this is valid in Coma, it would confirm that all the central spirals (see Fig. 2 of Paper I) have already gone through the cluster core, while the HI regular galaxies in the outskirts of the $\mathrm{X}$-ray emission are in the process of infalling (see Sect. 3). Some of the consequences in Coma are discussed in the next section. 
Table 2. HI and radio continuum parameters of abnormal spectrum galaxies (top) and blue disks (bottom) in Coma.

\begin{tabular}{|c|c|c|c|c|c|c|c|c|}
\hline ID & $\begin{array}{l}\text { Other name } \\
(2)\end{array}$ & $\begin{array}{l}\text { Morph. } \\
\text { type } \\
(3)\end{array}$ & $\begin{array}{c}M_{\mathrm{HI}} \\
10^{8} M_{\odot} \\
(4)\end{array}$ & $\begin{array}{r}F_{\text {cont }} \\
\text { mJy } \\
(5)\end{array}$ & $\begin{array}{c}\text { rms noise } \\
\text { mJy Beam }^{-1} \\
(6)\end{array}$ & $\begin{array}{c}L_{1.4} \\
10^{20} \mathrm{WHz}^{-1} \\
(7)\end{array}$ & $\begin{array}{c}S F R \\
M_{\odot} \mathrm{yr}^{-1} \\
(8)\end{array}$ & $\begin{array}{c}S F R / L_{\mathrm{B}} \\
M_{\odot}\left(\operatorname{yr} L_{\odot}\right)^{-1} \\
(9)^{a}\end{array}$ \\
\hline D 77 & Leda 83676 & $\mathrm{~S} 0 / \mathrm{a}(\mathrm{SB})$ & $<0.6$ & - & 0.18 & $<3.17$ & $<0.19$ & $<0.23$ \\
\hline D 94 & Leda 83682 & SA0 (PSB) & $<0.5$ & - & 0.18 & $<3.17$ & $<0.19$ & $<0.17$ \\
\hline D 112 & Leda 83684 & SB0 (PSB) & $<0.4$ & - & 0.18 & $<3.17$ & $<0.19$ & $<0.03$ \\
\hline D 21 & MCG 5-31-037 & SBa (PSB) & $<0.7$ & - & 0.19 & $<3.35$ & $<0.20$ & $<0.09$ \\
\hline D 73 & RB 183 & SA0 (PSB) & $<0.9$ & - & 0.17 & $<3.00$ & $<0.18$ & $<0.27$ \\
\hline D 44 & KUG1256+278A & So (SB) & $<0.6$ & - & 0.18 & $<3.17$ & $<0.19$ & $<0.18$ \\
\hline D 43 & NGC 4853 & SA0 (SB) & $<0.5$ & 1.2 & 0.18 & 7.06 & 0.42 & 0.06 \\
\hline D 89 & IC 3949 & SA0 (PSB) & $<0.3$ & 2.1 & 0.18 & 12.35 & 0.73 & 0.18 \\
\hline D 127 & RB 042 & S0 (PSB) & $<0.6$ & 1.0 & 0.18 & 5.88 & 0.35 & 0.46 \\
\hline D 216 & RB 160 & $\mathrm{Sa} \quad(\mathrm{PSB})$ & $<0.7$ & - & 0.18 & $<3.17$ & $<0.19$ & $<0.28$ \\
\hline D 99 & Mrk 060 & SB0 (PSB) & $<0.7$ & 1.2 & 0.19 & 7.06 & 0.42 & 0.23 \\
\hline D 146 & $\mathrm{RB} 110$ & So (PSB) & $<0.3$ & - & 0.18 & $<3.17$ & $<0.19$ & $<0.16$ \\
\hline D 61 & CGCG 160-104 & SA0 (PSB) & $<0.7$ & - & 0.10 & $<1.76$ & $<0.10$ & $<0.03$ \\
\hline D 189 & Leda 83763 & S0 (PSB) & $<1.5$ & - & 0.17 & $<3.00$ & $<0.18$ & $<0.31$ \\
\hline $160-055$ & NGC 4848 & Scd & 4.3 & 16.6 & 0.18 & 97.37 & 5.75 & 0.38 \\
\hline $160-073$ & Mrk 058 & $\mathrm{Sb}$ & 2.0 & 5.5 & 0.17 & 32.10 & 1.89 & 0.56 \\
\hline $160-086$ & & $\mathrm{Sb}$ & 1.7 & 3.8 & 0.15 & 22.11 & 1.30 & 0.88 \\
\hline $160-252$ & IC 4040 & $\mathrm{Sdm}$ & 3.3 & 15.0 & 0.18 & 88.20 & 5.20 & 1.00 \\
\hline $160-098$ & & $\mathrm{Sbc}$ & 7.3 & 5.7 & 0.18 & 33.34 & 1.97 & 0.62 \\
\hline $160-106$ & NGC $4926-\mathrm{A}$ & $\mathrm{Sa}$ & 6.0 & 3.1 & 0.15 & 18.17 & 1.07 & 0.49 \\
\hline
\end{tabular}

${ }^{a}$ In units of $10^{-9}$

\section{5. $\mathrm{H} I$ and radio continuum of PSB and active galaxies}

The physical mechanisms which trigger and quench the star formation activity, and the question if the star burst exhausts the Hi reservoir is still a matter of debate. We address this problem pointing at 14 galaxies in Coma for which abnormal spectra are reported by Caldwell et al. $(1993,1997)$, in order to get Hi and radio continuum information. The radio continuum at $1.4 \mathrm{GHz}$ could be due to synchrotron emission from relativistic electrons accelerated by supernovae hence probing star formation, or it could come from AGN. At intermediate redshift Smail et al. (1999) detect in the cluster CL 0939+4713 ( $z=0.41)$ several post starburst galaxies in radio continuum and these authors argue that the PSBs are most likely dust enshrouded starbursts. In the local universe Chang et al. (2001) observed in Hi five of the spectroscopically selected sample of E+A galaxies (Zabludoff et al. 1996). They detected one in HI and none in radio continuum. The implied low star formation rates and limits on the SFR for these galaxies rule out that any of these is in fact a dust enshrouded starburst.

Eleven of the 14 abnormal spectrum galaxies we pointed at in Coma are defined as PSBs because of their spectra with strong absorption lines and no emission at all; the three remaining are star forming systems (SBs), as indicated by their emission line spectrum superposed to absorption lines (C93). Their position relative to the X-ray emission is shown in Paper I (Fig. 6). We detected none of these objects in HI, and report very low Hi mass upper limits between 3 and $7 \times 10^{7} M_{\odot}$ (see Table 2). We do detect weak radio continuum emission in three PSB (IC 3949, Mrk 060 and RB 042) and one SB galaxy (NGC 4853, see Table 2). Our radio continuum levels in Coma imply even lower limits to the star formation rates than found by Miller \& Owen (2001) and
Chang et al. (2001) for the field E+A's. Thus in Coma, contrary to in CL $0939+4713$, the post starburst galaxies have at best only very modest levels of star formation. Chang et al. (2001) detected one of five E+A's in Hi, while our HI upper limits for the cluster PSBs are almost two orders of magnitude below this detection. This may indicate a real difference between cluster and field post starburst galaxies. Whatever triggers the starburst, whether collisions or ICM interaction, in a cluster the gas is cleaned out after the starburst, something that would not happen in the field. We show that the post starburst galaxies in Coma have very little, if any, Hi.

Table 2 gives in the upper part the Hi mass upper limits and radio continuum parameters for the sample of abnormal spectrum galaxies that we observed. For comparison, we show in the bottom the same results for the blue disks. RB 042 is projected very close to the $\mathrm{cD}$ galaxy NGC 4874 and was only marginally detected in radio. Three of four galaxies are new detections, the fourth, NGC 4853, is catalogued in the FIRST Survey (Becker et al. 1995). Table 2 gives in Cols. 1 and 2 the galaxy identification, Col. 3 gives the morphological and spectral classification, following C93. In Col. 4 we list the Hi upper limits, in Col. 5 the radio continuum flux, Col. 6 lists the rms noise in the continuum images. In Col. 7 we give the radio power, with upper limits set at 3 sigma. The associated SFR, listed in Col. 8, is calculated following Yun et al. (2001): $S F R_{1.4}=L_{1.4}\left[5.9 \times 10^{-22} \mathrm{~W} \mathrm{~Hz}^{-1}\right] M_{\odot} \mathrm{yr}^{-1}$. In Col. 9 we give the relation between the $S F R$ and the blue luminosity $\left(L_{\mathrm{B}}\right)$ obtained from the blue total magnitude, using the Mean Data from the LEDA database for homogeneity.

Table 2 shows that the $S F R$ in the PSBs is well below $1 M_{\odot} \mathrm{yr}^{-1}$. It comes as somewhat of a surprise that also the SB galaxies have $S F R$ less than $1 M_{\odot} \mathrm{yr}^{-1}$. This contrasts sharply with the blue disk galaxies reported by Bothun \& Dressler (1986), which were all detected in 
continuum (see Table 2) and for which the associated $S F R$ are from 1.0 to $5.5 M_{\odot} \mathrm{yr}^{-1}$. We note that these rates are in good agreement with the rates reported from the $\mathrm{H} \alpha$ equivalent widths by Bothun \& Dressler (1986). Thus the blue "starforming" disks have a significantly higher $S F R$ than the "starbursting" galaxies identified by C93. This is also valid if we consider the factor $S F R / L_{\mathrm{B}}$ given in Col. 9 of Table 2, which is probably a better criteria than merely $S F R$ if the sampled galaxies span a large range in size and luminosity. There is a consistent trend between Cols. 8 and 9 , with blue disks showing systematically higher values of $S F R / L_{\mathrm{B}}$ than galaxies from C93. The explanation is simple: C93 only selected early type galaxies for their sample of abnormal spectrum galaxies. The emission lines typically have narrow $\mathrm{H} \delta$ lines $(\leq 3 \AA)$, while the blue disks have $3 \AA \leq \mathrm{H} \delta \leq 7 \AA$. Thus the $\mathrm{SB}$ in the C93 sample means a higher than usual $S F R$ for an early type galaxy; the absolute levels are very low. It is interesting that the C93 sample is spatially distinct from the blue disks. The latter are located at the edge of the X-ray emission, while most of the C93 galaxies are in the cluster core or in the zone between the core and the SW group. The difference in environment may well account for the different star formation properties of these galaxies.

HST images (Caldwell et al. 1999) show that some of the PSB galaxies in Coma have retained their disk, even with a smooth spiral structure. D61 (Zw 160-104) shows two dust lanes forming an edge-on disk, and D216 (RB 160) shows clear spiral structure. Interestingly our limits to the $S F R$ and Hi content for these two galaxies are very low. Obviously the time scale for gas removal from these galaxies was shorter than for a morphological transformation. Caldwell et al. (1999) suggest that star formation activity in the PSBs of Coma could be triggered by galaxy harassment and by gravitational perturbations between the main cluster and the SW group. However, Moore et al. (1996) estimate a time-scale of $\sim 3$ Gyr to produce considerable changes in the optical morphology by this process. This is too long for galaxies like D 61 and D 216 to explain the whole process of triggering a starburst, the loss of a big fraction of their gas reservoir, and the subsequent quenching of the activity. An alternative process to explain this scenario is the re-accretion of gas mass after the stripping, as the infalling gas colliding with the clouds remaining in the disk can trigger star formation within the disk. In a cluster like Virgo this process starts at $\sim 2 \times 10^{8}$ years after the closest passage of the cluster core, and ends at $\sim 5 \times 10^{8}$ years (Vollmer et al. 2001b). The same process in Coma should develop with at least the same time-scales (or shorter, if we consider the more hostile ICM conditions), suggesting that the interaction with the ICM and gas re-accretion may account for this scenario. This is reinforced by the fact that galaxies could reach their present condition during a single pass through the cluster core, on a time scale $\leq 1$ Gyr, which is much shorter than the time needed by galaxy harassment to modify the optical morphology. The cases of the PSBs D 61 and D 216 support this scenario: the former presents a very young starburst age of $0.5 \mathrm{Gyr}$, and the latter, recently reclassified as a spiral (Caldwell et al. 1999), should have harbored a significant amount of gas in the recent past. Both need a faster process than galaxy harassment to account for the sudden removal of the Hi reservoir and quenching of star forming activity.

The gas which has been accelerated to values below the escape velocity will accrete back to the galaxy at time scales of $2-7 \times 10^{8}$ years (Vollmer et al. 2001b). As a first approach in Coma we apply a conservative value of $5 \times 10^{8}$ years for the re-accretion time scale, i.e. the same value found by Vollmer et al. in Virgo. If we consider a typical galaxy moving across the Coma cluster at $v_{\text {rel }} \sim$ $1000 \mathrm{~km} \mathrm{~s}^{-1}$ (equivalent to the velocity dispersion) it will travel some $0.5 \mathrm{Mpc}$ before the gas accretes back on to the disk and triggers a burst of star formation. It is very interesting to associate this with the fact that a considerable fraction $(38 \%)$ of the star forming UV flux in Coma is produced in an annular region between $20^{\prime}$ and $30^{\prime}$, or $\sim 0.5 \mathrm{Mpc}$ from the cluster center (Donas et al. 1995), and that it is in this zone where most of the blue galaxies in Coma are located (Paper I). Taking into account their position relative to the cluster, NGC 4848 and most of the blue disk galaxies in Coma have had enough time after passing across the cluster core to re-accrete gas clouds and boost a major star formation event; this mechanism may in part account for the blue annulus observed in Coma which is also observed in high redshift clusters (Oemler 1992).

\section{Conclusions}

In this paper we present the Hi morphology and kinematics of the brightest spirals in Coma. We compare the HI morphology with numerical simulations on ram pressure stripping by the ICM. We derive star formation rates for a sample of post starburst and actively star forming galaxies, from deep continuum imaging obtained as a byproduct of the Hi observations. We conclude that the Hi morphology of the spirals in Coma, the location of the Hi deficient galaxies and the size of the HI disks are consistent with predictions of the effect of ram pressure stripping by the ICM.

Targeted observations of 11 of the 14 known PSB galaxies in Coma give $\mathrm{HI}$ upper limits between 3 and $7 \times 10^{7} M_{\odot}$ in HI. The star formation rates derived from (upper limits to) the radio continuum are less than $1 M_{\odot} \mathrm{yr}^{-1}$. Even the early type galaxies with abnormal emission lines (SB galaxies from Caldwell et al.) have $S F R$ well below $1 M_{\odot} \mathrm{yr}^{-1}$. Thus in Coma there is no evidence for the presence of the dust enshrouded starburst galaxies, which may have been found in clusters at intermediate redshift. We found additional observational evidence confirming a real difference between cluster and field post starbursts; galaxies in clusters would exhaust the gas after the starburst, something that is not always observed in the field. 
Acknowledgements. We are very grateful to E. Brinks for helping to improve this paper significantly. HBA thanks the DAEC of the Observatoire de Paris, the Astronomy Department of Columbia University, and the AOC of the NRAO, for support and hospitality during his visits. We used the Digital Sky Survey, produced at the Space Telescope Science Institute. We have made use of the Lyon-Meudon Extragalactic Database (LEDA) supplied by the LEDA team at the CRAL-Observatoire de Lyon (France). We used NED, the NASA/IPAC extragalactic database, operated for NASA by the Jet Propulsion Laboratory at Caltech. This work has in part been supported by NSF grant AST-97-17177 to Columbia University. We appreciate the suggestions done by an anonymous referee and the efficiency with which this paper passed through the whole evaluation procedure.

\section{Appendix A}

Individual Channel Maps (Presented in increasing Right Ascension Order) This appendix is only available in electronic form at http://www.edpsciences.org

\section{References}

Abadi, M. G., Moore, B., \& Bower, R. G. 1999, MNRAS, 308, 947

Amram, P., Le Coarer, E., Marcelin, M., et al. 1992, A\&AS, 94,175

Arnaud, M., Aghanim, N., Gastaud, R., et al. 2001, A\&A, 365, L67

Becker, R. H., White, R. L., \& Helfand, D. J. 1995, ApJ, 450, 559

Bekki, K. 1999, ApJ, 510, L15

Biviano, A., Durret, F., Gerbal, D., et al. 1996, A\&A, 311, 95

Bothun, G., \& Dressler, A. 1986, ApJ, 301, 57

Bravo-Alfaro, H., Cayatte, V., van Gorkom, J. H., \& Balkowski, C. 2000, AJ, 119, 580 (Paper I)

Briel, U. G., Henry, J. P., \& Bohringer, H. 1992, A\&A, 259, L31

Briel, U. G., Henry, J. P., Lumb, D. H., et al. 2001, A\&A, 365, L60

Caldwell, N., Rose, J. A., Sharpless, R. M., Ellis, R. S., \& Bower, R. G. 1993, AJ, 106, 473

Caldwell, N., \& Rose, J. A. 1997, AJ, 113, 492

Caldwell, N., Rose, J. A., \& Dendy, K. 1999, AJ, 117, 140

Casoli, F., Dickey, J., Kazès, I., et al. 1996, A\&AS, 116, 193

Cayatte, V., van Gorkom, J. H., Balkowski, C., \& Kotanyi, C. 1990, AJ, 100, 604
Cayatte, V., Kotanyi, C., Balkowski, C., \& van Gorkom, J. H. 1994, AJ, 107, 1003

Chang, T., van Gorkom, J. H., Zabludoff, A.I., Zaritsky, D., \& Mihos, C. 2001, AJ, 121, 1965

Colless, M., \& Dunn, A. M. 1996, ApJ, 458, 435

Donas, J., Milliard, B., \& Laget, M. 1995, A\&A, 303, 661

Dressler, A., Smail, I., Poggianti, B. M., et al. 1999, ApJS, 122, 51

Dressler, A. 1984, ARA\&A, 22, 185

Gavazzi, G. 1989, ApJ, 346, 59

Gavazzi, G., Garilli, B., \& Boselli, A. 1990, A\&AS, 83, 399

Gavazzi, G., Contursi, A., Carrasco, L., et al. 1995, A\&A, 304, 325

Giovanelli, R., \& Haynes, M. P. 1985, ApJ, 292, 404

Gunn, J. E., \& Gott, J. R. 1972, ApJ, 176, 1

Icke, V. 1985, A\&A, 144, 115

Kenney, J. D. P., \& Young, J. S. 1989, ApJ, 344, 171

Lavery, R. J., \& Henry, J. P. 1994, ApJ, 426, 524

Lavezzi, T. E., Dickey, J. M., Casoli, F., \& Kazès, I. 1999, ApJ, 117, 1995

Miller, N. A., \& Owen, F. N. 2001, ApJ, 554, L25

Mirabel, I. F., \& Sanders, D. B. 1988, ApJ, 355, 104

Moore, B., Katz, N., Lake, G., Dressler, A., \& Oemler, A. 1996, Nature, 379,613

Moore, B., Lake, G., Quinn, T., \& Stadel, J. 1999, MNRAS, 304,465

Neumann, D. M., Arnaud, M., Gastaud, R., et al. 2001, A\&A, 365, L74

Nulsen, P. E. J. 1982, MNRAS, 198, 1007

Oemler, A. 1992, in Clusters and Superclusters of Galaxies, ed. A. C. Fabian (Dordrecht:Kluwer), 29

Poggianti, B. M., Smail, I., Dressler, A., et al. 1999, ApJ, 518, 576

Smail, I., Morrison, G., Gray, M. E., et al. 1999, ApJ, 525, 609

Solanes, J. M., Manrique, A., García-Gómez, C., et al. 2001, ApJ, 548, 97

Stevens, I. R., Acreman, D. M., \& Ponman, T. J. 1999, MNRAS, 310, 663

van den Bergh, S. 1976, ApJ, 206, 883

van den Bergh, S., Pierce, M., \& Tully, R. B. 1990, ApJ, 359, 4

Vikhlinin, A., Forman, W., \& Jones, C. 1997, ApJ, 474, L7

Vollmer, B., Braine, J., Balkowski, C., Cayatte, V., \& Duschl, W. J. 2001a, A\&A, 374, 824

Vollmer, B., Cayatte, V., Balkowski, C., Duschl, W. J. 2001b, ApJ, in press [astro-ph/0107237]

Warmels, R. H. 1988, A\&AS, 72, 19

Wild, J. P. 1970, Aust. J. Phys., 23, 113

Yun, M. S., Reddy, N. A., \& Condon, J. J. 2001, ApJ, in press [astro-ph/0102154]

Zabludoff, A. I., Zaritsky, D., Lin, H., et al. 1996, ApJ, 466, 104 